\title{
Models and Solutions for Radio Irregularity in Wireless Sensor Networks
}

\author{
GANG ZHOU, TIAN HE, SUDHA KRISHNAMURTHY, JOHN A. STANKOVIC \\ Department of Computer Science \\ University of Virginia
}

\begin{abstract}
In this paper, we investigate the impact of radio irregularity on wireless sensor networks. Radio irregularity is a common phenomenon which arises from multiple factors, such as variance in $\mathrm{RF}$ sending power and different path losses depending on the direction of propagation. From our experiments, we discover that the variance in received signal strength is largely random; however, it exhibits a continuous change with incremental changes in direction. With empirical data obtained from the MICA2 and MICAZ platforms, we establish a radio model for simulation, called the Radio Irregularity Model (RIM). This model is the first to bridge the discrepancy between spherical radio models used by simulators and the physical reality of radio signals. With this model, we investigate the impact of radio irregularity on several upper layer protocols, including MAC, routing, localization and topology control. Our results show that radio irregularity has a relatively larger impact on the routing layer than the MAC layer. It also shows that radio irregularity leads to larger localization errors and makes it harder to maintain communication connectivity in topology control. To deal with these issues, we present eight solutions to deal with radio irregularity. We evaluate three of them in detail. The results obtained from both the simulations and a running testbed demonstrate that our solutions greatly improve system performance in the presence of radio irregularity.
\end{abstract}

Categories and Subject Descriptors: C.2.1 [Computer Communication Network]: Network Architecture and Design; I.6 [Computer Methodologies]: Simulation and Modeling

General Terms: Design, Algorithms, Measurement, Performance, Experimentation

Additional Key Words and Phrases: Sensor networks, wireless communication, radio irregularity, sending power, path loss, link asymmetry, packet loss, localization, topology control

\section{INTRODUCTION}

Radio irregularity is a common and non-negligible phenomenon in wireless sensor networks. It results in irregularity in radio range and variations in packet loss in different directions, and is considered as an essential reason for asymmetric links as viewed by upper layers in the protocol stack. Several empirical studies [Ganesan et al. 2002][Woo et al. 2003][Zhao and Govindan 2003][Cerpa et al. 2003] on the Berkeley mote platform have shown that the radio range varies significantly in different directions and the percentage of asymmetric links in a system varies depending on the average distance between nodes.

The impact of radio irregularity on protocol performance can be investigated through a running system. However, few researchers have actually pursued this direction, because of two reasons: First, the complexity and cost of performance evaluations on a running system escalate, when sensor networks scale up to thousands or more nodes. Second, repeatable results of radio performance are extremely hard to obtain from uncontrolled environments, hence leading to difficulties in system tuning and performance evaluation. As a result, simulation techniques are used 
as an efficient alterative to evaluate protocol performance. Unfortunately, most existing simulations do not take radio irregularity, a common phenomenon in wireless communication, into account. The spherical radio patterns assumed by simulators such as [Zeng et al. 1998] may not approximate real radio properties well enough and hence may lead to an inaccurate estimation of application performance.

Several researchers [Ganesan et al. 2002][Woo et al. 2003][Zhao and Govindan 2003] [Cerpa et al. 2003] have already shown extensive evidence of radio irregularity in wireless communication. Their main focus is to observe and quantify such phenomena. This paper is distinguished from the previous ones for the initiative in bridging the gap between spherical radio models used by simulators and the physical reality of radio signals. We first verify the presence of radio irregularity using empirical data obtained from MICA2 and MICAZ platforms. The results demonstrate that the radio pattern is largely random; however, it exhibits a continuous change with incremental changes in direction. Based on experimental data, a radio model for simulations, called the Radio Irregularity Model (RIM), is formulated. RIM takes into account both the anisotropic properties of the propagation media and the heterogeneous properties of devices.

With the help of the RIM model, we explore the impact of radio irregularity on MAC, routing, localization and topology control performance. Among the protocols we evaluate, we find that radio irregularity has a significant impact on routing, localization and topology control protocols, but a relatively small impact on the MAC protocols. We also find that location-based routing protocols, such as Geographic Forwarding (GF) [Karp 2000] perform worse in the presence of radio irregularity than on-demand protocols, such as AODV [Perkins and Royer 1999] and DSR [Johnson and Maltz 1996]. We propose several potential solutions to deal with radio irregularity in wireless sensor networks. We evaluate the Symmetric Geographic Forwarding solution in simulation, and implement the Asymmetry Detection Service as well as the Bounded Distance Forwarding solution in running systems with 27 60 MICA2 devices. Our results illustrate that our solutions do succeed in alleviating the performance penalties due to radio irregularity.

The rest of this paper is organized as follows: we briefly analyze the causes and impact of radio irregularity in Section 2. In Section 3, we describe experimental data collected from the Berkeley mote platform and make some general conclusions about radio irregularity. Based on these conclusions, we propose the RIM radio model in Section 4. We then use the RIM model in simulations to analyze the impact of radio irregularity on MAC protocols in Section 5, routing protocols in Section 6, localization protocols in Section 7 and topology control protocols in Section 8. Solutions to deal with radio irregularity are proposed and evaluated in Section 9. Finally, we conclude the paper in Section 10.

\section{ANALYSIS OF RADIO IRREGULARITY}

In this section, we first identify the causes of radio irregularity, and then briefly discuss the impact of irregularity on the different protocol layers.

\subsection{Causes of Radio Irregularity}

Radio irregularity is caused by two categories of factors: devices and the propagation media. Device properties include the antenna type (directional or omni- 
directional), the sending power, antenna gains (at both the transmitter and receiver), receiver sensitivity, and the Signal-Noise-Ratio (SNR) threshold. Media properties include the media type, the background noise and some other environmental factors, such as the temperature and obstacles within the propagation media.

In general, the radio irregularity is caused by the anisotropic properties of the propagation media and the heterogeneous properties of devices. Among all these factors, we focus on the anisotropic path losses and the differences in signal sending power, which are commonly regarded as the key causes of radio irregularity.

-Anisotropic Path Losses: The variance in the signal path loss is one of the major causes of radio irregularity. When a signal propagates within a medium, it may be reflected, diffracted, and scattered [Shankar 2001]. Reflection occurs when an electromagnetic signal encounters an object, such as a building, that is greater than the signal's wavelength. Diffraction occurs when the signal encounters an irregular surface, such as a stone with sharp edges. Scattering occurs when the medium through which the electromagnetic wave propagates contains a large number of objects smaller than the signal wavelength. The medium is normally different in different directions. Consequently, radio propagation exhibits anisotropic patterns in most environments.

Another significant reason for anisotropic path loss is hardware differences. A node may not have the same antenna gain along all propagation directions, possibly due to hardware manufacturing. Hence, the anisotropic antenna gain of each node also contributes to the anisotropic path loss.

- Heterogeneous Sending Powers: Sensor devices may transmit RF signal at different sending powers, even though they are the same kind of devices. This difference may arise from some random factors during the manufacture of sensor devices. In addition, after the sensor devices are deployed, the batteries of different sensor devices deplete at different rates, due to different workloads and different environments in which they are deployed. Heterogeneous sending powers result in variable communication ranges, and cause anisotropic connectivity.

Environment and hardware differences are the two major sources of radio irregularity, and we propose a radio model to simulate these two factors. We are also aware that there are methods to adjust for differences in output power, noise, and manufacturing differences. Readers can refer to [Whitehouse and Culler 2002][Hoff and Azuma 2000][Hightower et al. 2000] for details. Our focus of this paper is to simulate the hardware differences, rather than to calibrate hardware differences.

\subsection{Impact of Radio Irregularity}

Radio irregularity is a non-negligible phenomenon in wireless systems. It's an essential reason for asymmetric radio interference and asymmetric links in upper layers. It can directly or indirectly affect many aspects of upper layer performance.

Asymmetric radio interference between neighboring nodes affects the correctness of MAC layer functions. For example, in the presence of radio irregularity, a node might not be able to successfully reserve the wireless channel through RTS and CTS handshaking, because those neighboring nodes of the receiver, which cannot hear the CTS control packet, might disrupt the receiving node. So, radio irregularity 
increases the chance of channel reservation failure and reduces the delivery ratios of data frames at the MAC layer.

Radio irregularity can also affect the performance and even correctness of networking protocols such as [He et al. 2003][Johnson and Maltz 1996][Karp and Kung 2000][Karp 2000]. For example, link asymmetry is one of the ways in which radio irregularity manifests itself at the higher layer. Link asymmetry has an adverse impact on protocols that use path-reversal techniques to establish an end-to-end connection.

Actually, the impact of radio irregularity is not only confined to the MAC and routing layers, radio irregularity also influences other protocols, such as localization, sensing coverage and topology control protocols.

Localization protocols such as DV-HOP [Niculescu and Nath 2003] and Centroid [N. Bulusu and Estrin 2000] assume a spherical radio range. The study in [He et al. 2003] shows that the performance of such protocols degrades when the radio range becomes irregular. The sensing coverage scheme in [Yan et al. 2003] assumes that sensing and communication ranges are spherical. In the presence of radio irregularity, they might not be able to guarantee full coverage and blind areas would occur. The topology control scheme in GAF [Xu et al. 2001] builds a communication mesh based on the assumption of a spherical range. This might lead to network partition in the presence of a non-spherical range. We note that some other topology control protocols, such as ASCENT [Cerpa and Estrin 2002] and Span [Chen et al. 2001] do not depend on such an assumption, however, performance evaluations of those protocols considering radio irregularity would be interesting future work.

In the rest of the paper, we evaluate the impact of radio irregularity on many upper layer protocols, including the MAC layer, the routing layer, localization protocols and topology control protocols.

\section{RADIO IRREGULARITY IN REALITY}

We conduct several experiments ${ }^{1}$ to study the irregularity of the radio using MICA2 motes, and in this section we discuss some of the experimental results we obtain from an outdoor environment. Our results confirm that radio propagation is largely anisotropic and exhibits a continuous variation with incremental changes in direction.

\subsection{Experimental Setup}

We use a pair of MICA2 motes for our experiments. One of the motes periodically transmits probing beacons and the other mote samples its ADC port while receiving these beacons. The ADC reads the signal on the analog pin of the Chipcon transceiver [ChipconCC1000 ] and converts it into a 10-bit voltage value. The voltage reading is mapped into the received signal strength in $\mathrm{dBm}$ according to the specification in [ChipconCC1000 ]. All experiments are conducted in an open parking lot near a building, and all devices are equipped with whip antennae with the length of a quarter of the radio $(433 \mathrm{MHz})$ wavelength.

\footnotetext{
${ }^{1}$ This work is proposed to study and simulate the degree of radio irregularity, not the exact radio pattern. Readers can refer to [RF Chamber] on how to use an RF chamber to measure highly accurate radio patterns in special labs.
} 


\subsection{Experimental Results}

In this section, we demonstrate the presence of radio irregularity using three different metrics: 1) the received signal strength, 2) the packet reception ratio and 3) the communication range.

3.2.1 Anisotropic Signal Strength. In the first experiment, the receiver is placed 10 feet away from the sender (both on ground) and the received signal strength is measured in four different geographical directions by sampling 100 beacons received in each direction.

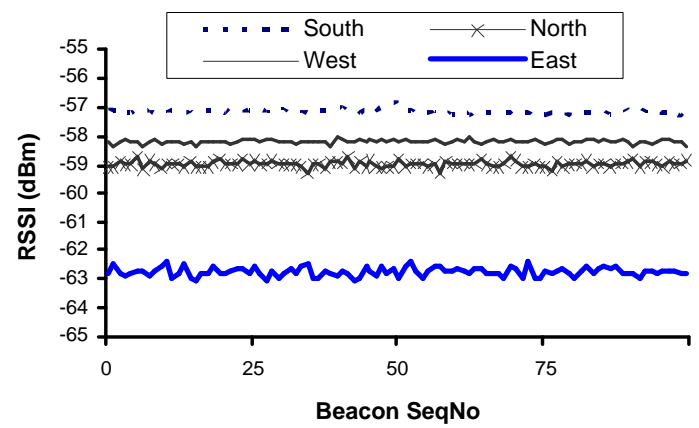

Fig. 1. Signal Strength over Time in Four Directions

Figure 1 shows that the received signal strength in each direction is relatively stable over time (The small variance comes from the fading effect [Shankar 2001]). However, the signal strength received in the south is much higher than that received in the east, although nodes have the same distance from the sender. We also measure the variation of signal strength with the changes in the angular direction of the receiver with respect to the sender. Figure 2 shows the variation of the received signal strength as a function of the angular direction with respect to the sender, when the distance between the sender and receiver is 10 feet and 20 feet, respectively. These results show that the received signal strength varies continuously $^{2}$ with the direction. In other words, incremental changes in direction result in incremental variation in the received signal strength.

3.2.2 Anisotropic Packet Loss Ratio. Figure 3 shows how the packet reception ratio varies in different directions. When the sender and receiver are placed 10 feet apart, the packet reception ratio is nearly $100 \%$ in all the directions, because the signal is still strong in all the directions. However, when they are placed 20 feet apart, there is a $90 \%$ packet loss in the east direction. This result is consistent with the results shown in Figure 1, which demonstrates that the received signal strength measured in the east is lower than that in the other three directions.

$\overline{{ }^{2} \text { We call the }}$ variation continuous if and only if the maximum received signal strength percentage variance per unit degree change in the direction of radio propagation is smaller than 0.05 . 


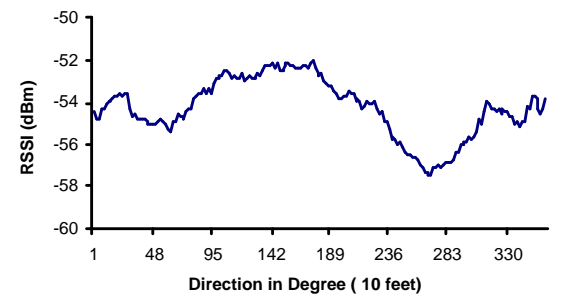

(a) Measured at 10 feet

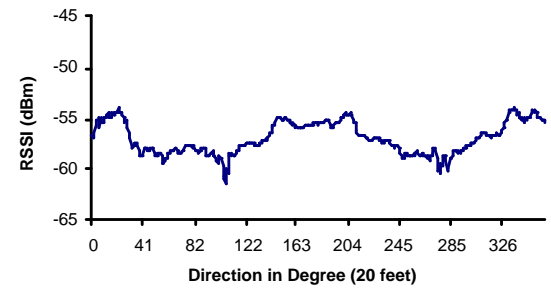

(b) Measured at 20 feet

Fig. 2. Signal Strength Values in Different Directions

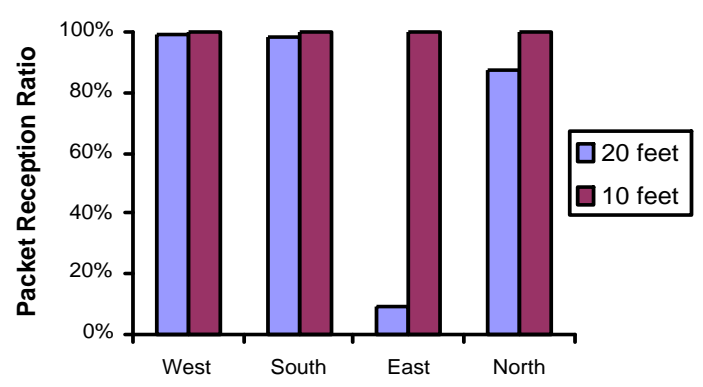

Fig. 3. Anisotropic Packet Reception

3.2.3 Anisotropic Radio Range. Another aspect in which we demonstrate the irregularity is to show that the communication range of a mote is not uniform in all directions. In the experiment, we fix the received signal strength threshold at $-55.5 \mathrm{dBm}$ and $-59 \mathrm{dBm}$, respectively. Then with such thresholds, we measure the communication ranges in different directions. Figure 4 shows the communication range of a mote as the receiver direction varies from degree 0 to degree 359 . The range map shown in Figure 4 is another confirmation of radio irregularity in a wireless medium.

3.2.4 Range Irregularity with Varying Sending Power. We also investigate the received signal strength when the sending power varies due to different battery status and hardware differences. In Figure 5(a), we use the same sender and receiver, placed 10 feet apart. We change the batteries at the sender side each time. The result indicates that different battery status at the same sender can affect the received signal strength. In Figure 5(b), we use the same batteries, but in different senders each time. The same receiver is used, placed 10 feet apart from the sender. The result shows that different senders with the same batteries can also affect the received signal strength. 


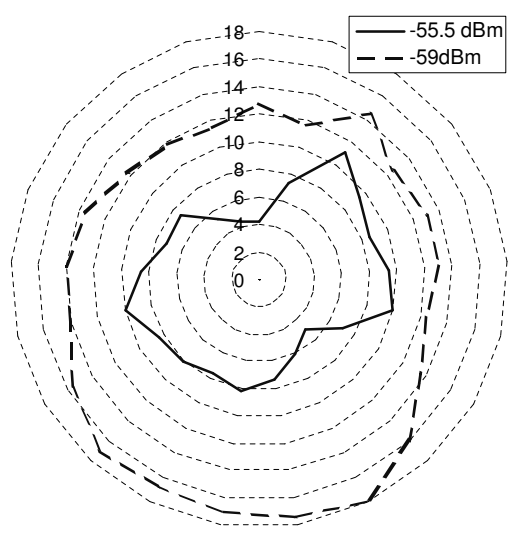

Fig. 4. Anisotropic Range

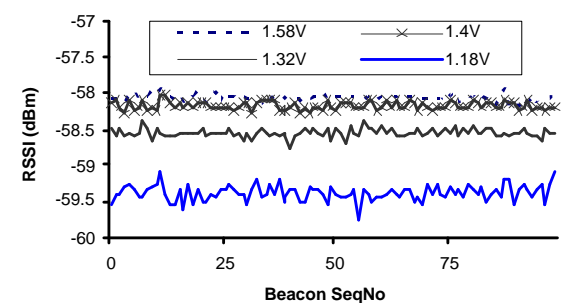

(a) One mote with different battery status

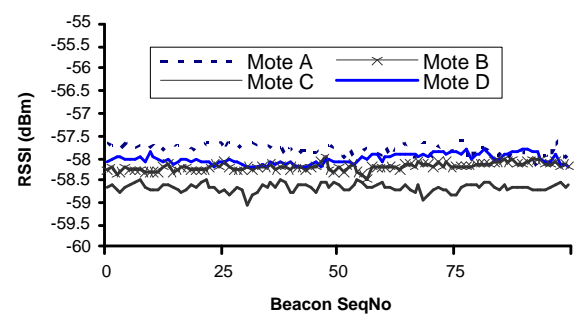

(b) Different motes with the same battery status

Fig. 5. Radio Irregularity with Sending Powers

\subsection{Summary of Experimental Results}

From the experimental results, we infer that the radio of sensor devices has the following main properties:

(1) Anisotropy: The radio signal from a transmitter has different path losses ${ }^{3}$ in different directions (Figure 1 and Figure 2).

(2) Continuous variation: The signal path loss varies continuously with incremental changes of the propagation direction from a transmitter (Figure 2 and Figure 4).

(3) Heterogeneity: Differences in hardware property and battery status lead to different signal sending powers, hence different received signal strengths (Figure 5).

\footnotetext{
${ }^{3}$ Figure 2 shows that the received signal strength varies greatly in different propagation directions, while Figure 1 tells us that the fading effect does not cause much variation in the received signal strength for a specified direction. Accordingly, it is reasonable to believe that different path losses in different directions are the main reason for the received signal strength variations in different propagation directions.
} 


\section{MODELING RADIO IRREGULARITY}

As we have shown in our experiments as well as demonstrated in other research results [Ganesan et al. 2002][Woo et al. 2003][Zhao and Govindan 2003][Cerpa et al. 2003], radio irregularity is a common phenomenon in wireless sensor networks. Therefore, it is essential for simulations of wireless systems to capture such effects. This section describes our effort to model such a phenomenon in simulation environments.

\subsection{Isotropic Radio Models}

In isotropic radio models, the received signal strength is usually represented with the following formula:

$$
\text { Received Signal Strength }=\text { Sending Power }- \text { Path Loss }+ \text { Fading }
$$

The SendingPower of a node is determined by the battery status and the type of transmitter, amplifier and antenna. PathLoss describes the signal's energy loss as it travels to the receiver. Many models are used to estimate the PathLoss, such as the free-space propagation model, the two-ray model and the Hata model [Shankar 2001]. All these models are isotropic, meaning that the signal attenuates exactly the same in all directions. However, our experience as well as results obtained by others [Ganesan et al. 2002][Woo et al. 2003][Zhao and Govindan 2003][Cerpa et al. 2003] all indicate that the isotropic models do not hold well in practice.

\subsection{Radio Irregularity Model (RIM)}

The RIM model we propose here is an extension to isotropic radio models. It enhances isotropic radio models by approximating three main properties of radio signals: anisotropfy, continuous variation and heterogeneity, as we summarized in Section 3.3. These properties are normally ignored by previous isotropic radio models.

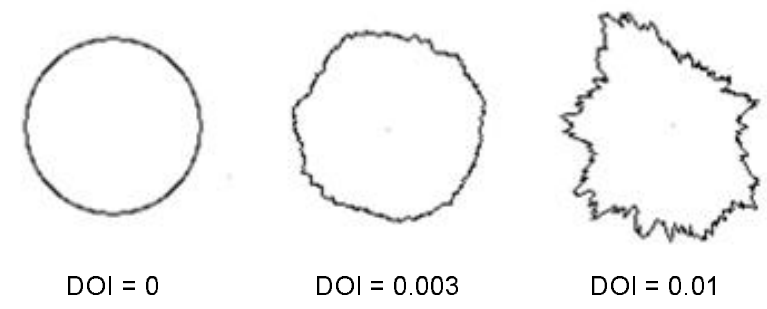

Fig. 6. Degree of Irregularity

To denote the irregularity of a radio pattern, the parameter DOI ( degree of irregularity) is introduced into the RIM model. The DOI parameter is defined as the maximum path loss percentage variation per unit degree change in the direction of radio propagation. As shown in Figure 6, when the DOI is set to 0, there is no range variation, and the communication range is a perfect sphere. However, when we increase the DOI value, the communication range becomes more and more irregular. 


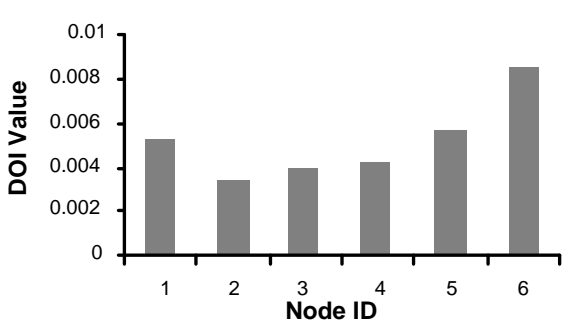

(a) A Short Antenna MICA2 System

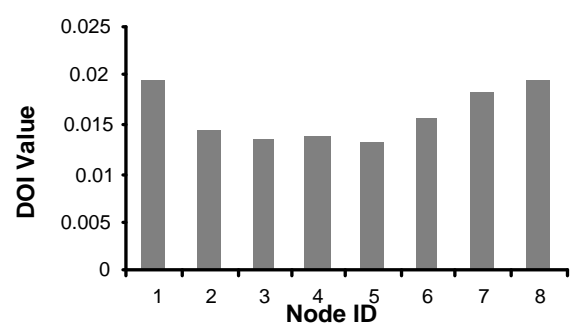

(b) A Long Antenna MICA2 System

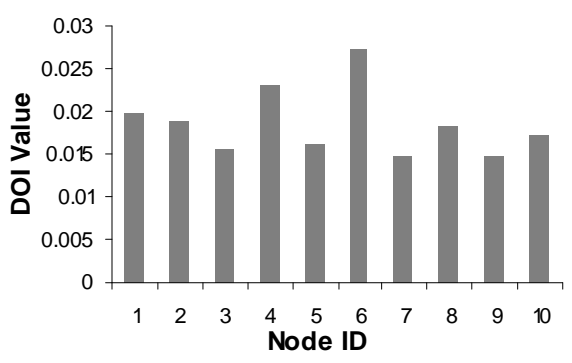

(c) A MICAZ System

Fig. 7. DOI Values from MICA2 Experiments

The RIM model is a general radio model which can default to the isotropic model when the DOI value is 0. The RIM model is established based on data from real sensor devices. It is a hybrid approach, which introduces real data (DOI value) into simulations, so that the radio irregularity pattern in reality can be approximated well. We repeat the experiments shown in Figure 2 on 6 MICA2 devices in a vehicle tracking system, and calculate the corresponding DOI values, according to the DOI definition. The experimental results depicted in Figure 7(a) inform that the variances of the received signal strength with incremental changes in directions are small, which validates our conclusion about continuous variation.

In order to investigate possible DOI variance in different types of devices, we repeat the experiments with new MICA2 motes that have double length antennae, $1 / 2$ the radio wavelength. Each node's DOI value is calculated and presented in Figure 7(b), which shows comparatively larger DOI values than those in Figure 7(a). This is because the new MICA2 devices have longer and more powerful antennae that amplify existing hardware differences.

To explore the RIM model's applicability across platforms and radios, we repeat the experiments with MICAZ [CROSSBOW ] motes, each of which has a whip antenna of $1 / 4$ radio wavelength. MICAZ motes use the $\mathrm{CC} 2420$ radio [ChipconCC2420 ], which follows the IEEE 802.15.4 [IEEE 802.15.4 1999] standard and is different from the CC1000 [ChipconCC1000] radio used in MICA2 motes. As Figure 7(c) illustrates, the RIM model applies in MICAZ devices and the measured DOI values have the range from 0.015 to 0.03. Comparing Figure 7(a) with Figure $7(\mathrm{c})$, we observe that MICAZ motes exhibit a higher degree of radio irregularity than MICA2 motes, when both types of devices use $1 / 4$ wavelength whip antennae. This is because the CC2420 radio in MICAZ is more powerful than the CC1000 
radio in MICA2.

4.2.1 Anisotropy Property in the RIM Model. Many models are used to estimate path loss, such as the free-space propagation model, the two-ray model and the Hata model [Shankar 2001]. These models are isotropic in the sense that the path losses in different directions are the same. To reflect the two main properties of radio irregularity, namely anisotropy and continuous variation, we adjust the value of path loss models in Equation 1 based on DOI values, resulting in the following formula:

$$
\begin{array}{r}
\text { Received Signal Strength }=\text { Sending Power }- \text { DOI Adjusted Path Loss }+ \text { Fading } \\
\text { where } \quad \text { DOI Adjusted Path Loss }=\text { Path Loss } \times K_{i}
\end{array}
$$

Here $K_{i}$ is a coefficient to represent the difference in path loss in different directions ${ }^{4}$. Specifically, $K_{i}$ is the $i^{\text {th }}$ degree coefficient, which is calculated as follows:

$$
\begin{aligned}
K_{i}= \begin{cases}1 & \text { if } i=0 \\
K_{i-1} \pm \text { Rand } \times D O I & \text { if } 0<i<360 \wedge i \in N\end{cases} \\
\text { where }\left|K_{0}-K_{359}\right| \leq D O I
\end{aligned}
$$

We can generate $360 K_{i}$ values for the 360 different directions, based on Equation 3 , by randomly fixing a direction as the starting direction represented by $i=0$. For the direction which does not have an integer value of angle from the start direction, we interpolate the $K_{i}$ value based on the values of the two adjacent directions which have integer angles from the starting direction.

$$
\begin{aligned}
K_{i}= & K_{s}+(i-s) \times\left(K_{t}-K_{s}\right) \\
& \text { where } s=\lfloor i\rfloor \wedge t=\lceil i\rceil \bmod 360 \wedge 0<i<360 \wedge i \notin N
\end{aligned}
$$

The statistical analysis of our experimental data indicates that the variance of received signal strength (mainly because of path loss variation since Figure 1 shows that fading is pretty small) in different directions fits the Weibull [Devore 1982] distribution. The Weibull distribution can be used to model natural phenomena such as variation of wind speed, scattering of radiation, etc. The Rayleigh distribution, which is commonly used for modeling multi-path fading in wireless communication, is a special case of the Weibull distribution. Analysis details are provided in Appendix A. In Equation 3, we generate a random number according to the Weibull distribution.

We conduct experiments to evaluate the RIM model's ability to generate radio patterns that have specified degree of radio irregularity. We input to the RIM model the degree of irregularity value $\mathrm{DOI}=0.01821^{5}$, which is measured in a MICA2 device and illustrated as the value of column 7 in Figure 7(b). Then the

\footnotetext{
${ }^{4}$ Coefficient $K_{i}$ is used to adjust the path loss in a specified direction. In this specified direction, we can also propose to adjust $K_{i}$ 's value in a small range based on the distance the receiver is from the transmitter, because when the distance increases, the signal travels in a larger environment area. So it may suffer different reflection, diffraction and scattering and has different path loss. We leave this as future work.

${ }^{5}$ Here, we keep 5 digits after the point to illustrate how accurate the RIM model is in simulating the degree of radio irregularity. As shown in later sections, maintaining 3 digits after the point is good enough for performance evaluation.
} 
DOI values of generated radio patterns from the RIM model are calculated and compared with the input DOI value. Figure 8 presents the result.

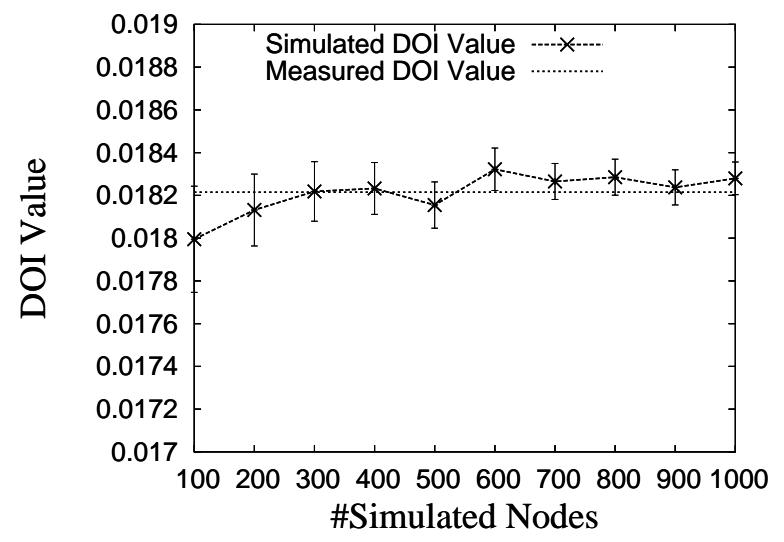

Fig. 8. Degree of Irregularity Evaluation (with 90\% Confidence Intervals)

As Figure 8 illustrates, the RIM model is very accurate in simulating the degree of radio irregularity. When the simulation uses 100 nodes, the length of the $90 \%$ confidence interval is less than 0.0005 , which is only $2.7 \%$ compared with the input DOI value 0.01821. In addition, according to performance evaluation (Figures $12,18,21,22,23$ and 25 ) in later sections of this paper, 0.0005 is too small to distort the performance evaluation.

Moreover, with the increase of the sample space, the RIM model converges towards the target DOI value. For example, when simulation uses 1000 nodes, the length of the $90 \%$ confidence interval decreases to 0.00015 . Compared with the input value 0.01821 , it only has $0.8 \%$ variation. The RIM model also gives more accurate average DOI values with the increase in the number of simulated nodes. The average DOI is 0.01799 when 100 nodes are used, and it becomes 0.01827 when 1000 nodes are used, which is much closer to the target value 0.01821 .

Accordingly, statistically speaking, the RIM model has the ability to simulate the degree of radio irregularity.

4.2.2 Heterogeneity Property in the RIM Model. Due to different battery status and hardware differences, the received signal strength can be different from two sending nodes of the same type in the same experimental setting. In RIM, we use the variance of signal sending power to account for such a difference. We introduce the second parameter named VSP (Variance of Sending Power), which is defined as the maximum percentage variance of the signal sending power among different devices. The new signal sending power is modelled by the following equation:

$$
V S P \text { Adjusted Sending Power }=\text { Sending Power } \times(1+\text { Rand } \times V S P)
$$

In Equation 5, we assume that the variance of sending power fits the normal distribution, which is broadly used to estimate battery lifetime distribution [Battery Lifetime ] and to simulate hardware differences [Devore 1982]. 
With the two parameters: DOI and VSP, the RIM model can be formulated as follows:

$$
\begin{aligned}
\text { Received Signal Strength }= & \text { VSP Adjusted Sending Power } \\
& - \text { DOI Adjusted Path Loss }+ \text { Fading }
\end{aligned}
$$

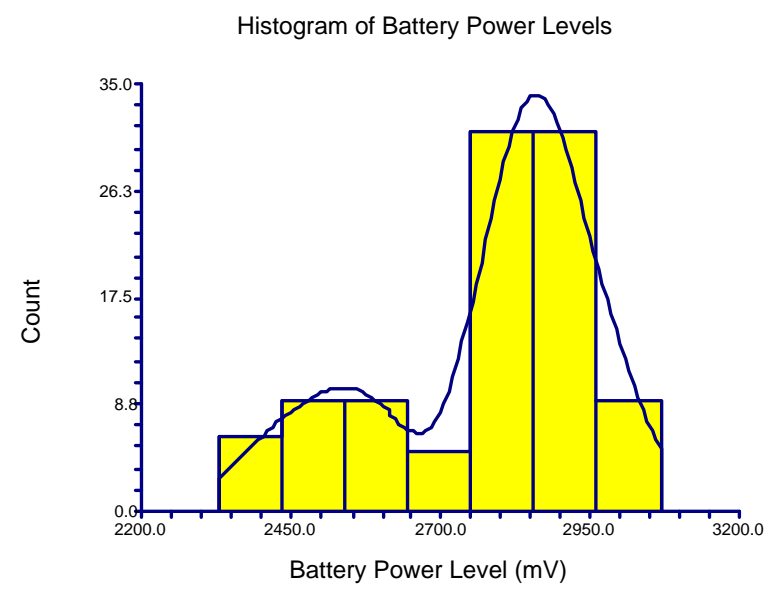

Fig. 9. Battery Power Level Snapshot in the VigilNet System

We are also aware that the default normal distribution we implemented in RIM is not a universal solution for all sensor network systems. The normal distribution may work well for initially deployed systems, which are equipped with new batteries. However, with respect to a system that has been used for a long time, the battery power level may not fit the normal distribution.

We take a snapshot of all battery power levels in the VigilNet System [He et al. 2004], and plot the distribution in Figure 9.

As shown in Figure 9, the battery power level does not fit a normal distribution well. This is because in the system design, all nodes are divided into two groups: sentry nodes and non-sentry nodes. Sentry nodes are supposed to work all the time and non-sentry nodes are put to sleep to save power. Non-sentry nodes are only awakened when an important event happens. Accordingly, this sentry design leads to more energy consumption for sentry nodes and less energy consumption for nonsentry nodes. To simulate this non-normal distribution, readers are encouraged to replace the normal random number generator in the RIM model with their own random number generators, to reflect the power level distribution reality in their own systems, and there is no need to modify any other code in RIM.

4.2.3 DOI Variance in a System. From empirical data we collected in two MICA2 systems and a MICAZ system shown in Figure 7, we observe that sensor devices in a system may have different DOI values, depending on the hardware devices used and the deployment environment. It is not convenient to measure each node's DOI value in a large scale system and assign the measured DOI values to each node in simulation. In order to reflect this fact of DOI variance among 
different devices in a system, we introduce the third parameter VDOI (Variance of DOI), which is defined as the maximum percentage variance of DOI values among different devices in a system. We assume the DOI variance in a system fits the normal distribution. So with the distribution as well as the VDOI value, each node in the system can easily get a DOI value. In performance evaluation of this paper, we first set VDOI as 0 to observe system performance with different DOI values, and then set VDOI greater than 0 to investigate performance sensitivity to different VDOI values.

\subsection{Comparison with a Binary DOI Model}

The RIM model is motivated by a simple binary DOI (Degree of Irregularity) model briefly mentioned in the localization work [He et al. 2003]. In the binary DOI model, the DOI parameter was originally defined as the maximum range variation per unit degree change in the direction of radio propagation.

The DOI model assumes an upper and lower bound on signal propagation, which are depicted as the inner and outer dashed circles in Figure 10(a). Beyond the upper bound, all nodes are out of communication range; and within the lower bound, every node is guaranteed to be within the communication range. If the distance between a pair of nodes is between these two boundaries, three scenarios are possible: 1) symmetric communication, 2) asymmetric communication, and 3) no communication.

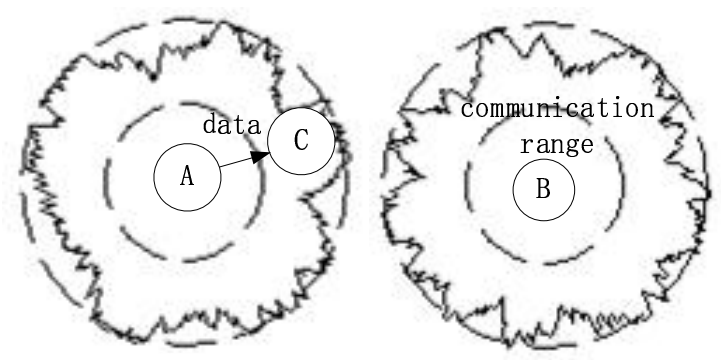

(a) No interference in the binary DOI model

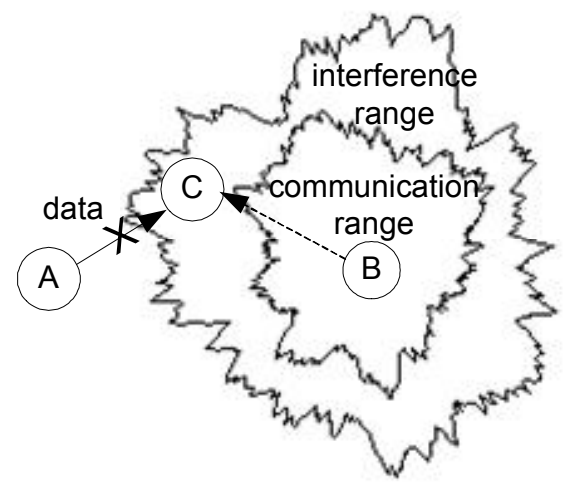

(b) Interference in the RIM model

Fig. 10. Communication Interference 
The binary DOI model is a good start to model signal irregularity. However, it does not model interference in real devices well. Since the DOI model is based on an absolute communication range, it assumes that within the inner range, the signal is very strong and can always be received correctly, while beyond the outer range there is no signal at all. This binary pattern is not true in reality. For example, in Figure 10(a), the DOI model assumes that there is no interference between nodes $\mathrm{B}$ and $\mathrm{C}$.

However in reality, there are no such clear boundaries and the communications of nodes do interfere with each other. Different from the binary DOI model, the RIM model we propose takes the radio sending energy, the energy loss, the background noise, and the interference among different communication signals into account.

The difference can be further explained with an example. In Figure 10(b), the RIM model allows node B's signal to propagate beyond its communication range to reach node $\mathrm{C}$, even though it is not strong enough for node $\mathrm{C}$ to receive it as a valid packet. This weak signal from node $\mathrm{B}$ acts as one source of background noise around node $\mathrm{C}$. In this case, node $\mathrm{C}$ may not be able to receive packets from node A, if the received signal is not stronger than the product of the Signal-Noise-Ratio (SNR) threshold and background noise level of node C.

The DOI model only models an absolute range based on the distance and determines whether one node can hear another node only by comparing the distances between these two nodes with the sender's communication range. With such a binary decision, it can't deal with interference as we mentioned earlier.

The RIM model enhances the isotropic radio model and the DOI radio model, by combing the energy models and the DOI factor together. The original DOI concept is redefined by incorporating radio energy propagation. We note that RIM is a general radio model which can default to the isotropic model when the DOI value is 0. Also, it can default to the DOI model when there is no interference among nodes.

We need to clarify that the RIM model is not proposed to simulate the exact radio pattern. Instead, it is a general radio model to simulate the degree of radio irregularity. Given measured radio patterns from a real system, the values of DOI, VSP and VDOI can be calculated and configured in the RIM model. Then the RIM model can generate a specified number of radio patterns that have the same degree of radio irregularity. For a system that adopts hardware calibration schemes [Whitehouse and Culler 2002][Hoff and Azuma 2000][Hightower et al. 2000], the values for DOI, VSP and VDOI are configured smaller, according to the measured values from sampled devices, so that the reduced radio irregularity is simulated. In a similar way, the RIM model can be configured to simulate systems that consists of hardware with different transceivers.

The RIM model is proposed to account for the three main properties of radio irregularity: anisotropy, continuous variation and heterogeneity, as we summarized in Section 3.3. Currently, the experiments we present are conducted in a parking place with MICA2 and MICAZ devices. The exact radio patterns and the degree of radio irregularity may vary in different environments. We expect that the radio is more irregular in a harsher environment, such as in a wild forest. We also expect that the three main properties of radio irregularity still hold in a harsher environ- 
ment, and the radio irregularity can be simulated by choosing larger DOI values. This is subject to further confirmation with experiments in different environments.

In the following sections, we use the RIM model as a simulation tool to help explore the impact of radio irregularity on MAC, routing, localization and topology control protocols.

\section{IMPACT ON MAC LAYER}

In this section, we first analyze how operations in the MAC layer are affected by radio irregularity. We then quantify the degree of $\mathrm{MAC}$ performance degradation in the presence of radio irregularity.

\subsection{Logical Analysis of the Impact}

Most contention-based MAC protocols are based on carrier sensing or handshaking techniques. In this section, we analyze the impact of radio irregularity from the technical point of view.

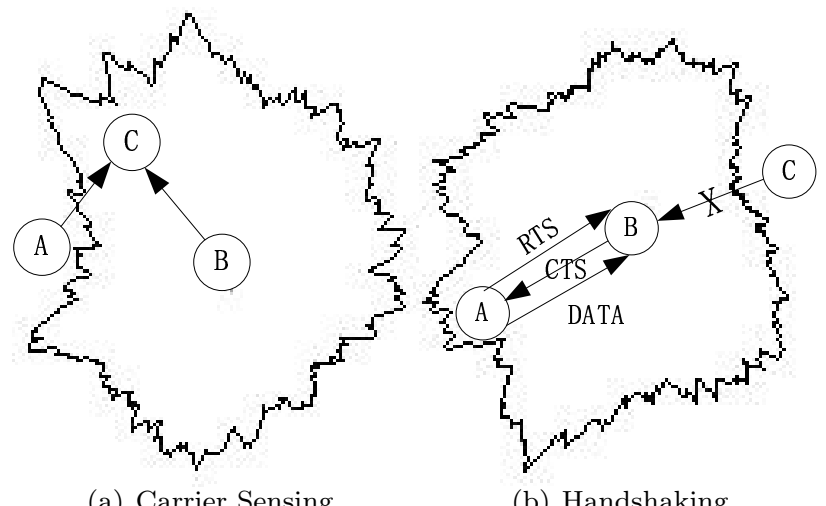

(a) Carrier Sensing

(b) Handshaking

Fig. 11. Impact on MAC Protocols

(1) Impact on Carrier Sensing: Radio irregularity increases the chance for MAC protocols that use the carrier sensing technique to get involved in the hidden terminal problem. For example, in Figure 11(a), while node B is transmitting packets to node $\mathrm{C}$, due to the irregularity, node $\mathrm{A}$ cannot detect the signal from node B, so node A senses a clear channel and starts to transmit packets. As a result, a collision happens at receiver $\mathrm{C}$. This scenario does not occur if node $\mathrm{B}$ has a spherical radio range that covers node $\mathrm{A}$ so that $\mathrm{A}$ can sense node B's signal and will not send a packet to $\mathrm{C}$ and get corrupted. Typical protocols using the carrier sensing technique are CSMA [Kleinrock and Tobagi 1975], MACA [Karn 1990], MACAW [Bharghavan et al. 1994] and 802.11 DCF [IEEE 802.11 1999].

(2) Impact on handshaking: The handshaking technique is specially designed to resolve hidden and exposed terminal problems. However, they cannot resolve the hidden and exposed terminal problems due to asymmetry, which can be 
Table I. Simulation Configuration One

\begin{tabular}{||l|l||}
\hline TERRAIN & $(150 \mathrm{~m} \mathrm{X} \mathrm{150m)}$ \\
\hline Node Number & 100 \\
\hline Node Placement & Uniform \\
\hline Application & Many-to-one CBR Streams \\
\hline Payload Size & 32 Bytes \\
\hline Routing Protocol & AODV, DSR, GF \\
\hline MAC Protocol & CSMA, 802.11 (DCF) \\
\hline Radio Layer & RADIO-ACCNOISE \\
\hline Radio Model & RIM \\
\hline Nominal Radio Range & $40 \mathrm{M}$ \\
\hline Radio Bandwidth & $200 \mathrm{~Kb} / \mathrm{s}$ \\
\hline
\end{tabular}

produced by radio irregularity. This can be demonstrated in an example (Figure 11(b)). We assume that node A sends a RTS message to node B, and then node $\mathrm{B}$ responds with a CTS message to node A. Any node overhearing the CTS message is supposed to wait long enough for node A to send out the data packet. If node C can't hear the CTS message from node B while node B can hear node $\mathrm{C}$, there will be a collision if node $\mathrm{C}$ sends data. Similar examples can be found for the exposed terminal case.

\subsection{Quantitative Analysis of the Impact}

We implemented the RIM model in the radio layer of GloMoSim [Zeng et al. 1998], a scalable discrete-event simulator developed by UCLA. We first describe our simulation configuration, and then evaluate the performance impact under different DOI and different VSP values, respectively.

This is not a media access control paper and we do not try to explore the impact of radio irregularity on all MAC protocols. We choose two typical MAC protocols, CSMA and 802.11 DCF, for case study, because they are popular protocols and also very typical protocols that use carrier sensing and RTS-CTS handshaking techniques. Readers can refer [Ye et al. 2002][Rajendran et al. 2003][Dam and k. Langendoen 2003][Woo and Culler 2001][Polastre et al. 2004] for more MAC protocols.

In the experiments, we use six CBR streams as the workload and set the CBR rate at a low rate, in order to isolate the effect of congestion and radio irregularity. Two metrics are used: 1) the loss ratio (number of packets lost / number of packets sent) and 2) the average single hop delay of received packets. We vary the DOI and VSP values separately in order to isolate and identify the impact individually. In each data value we present, we also give the corresponding $90 \%$ confidence interval.

In order to make our evaluation close to existing hardware proposed for use in wireless sensor network environments [CROSSBOW ], we use the simulation configuration shown in Table I. In all experiments, we investigate the range of DOI values according to the experimental data obtained from MICA2 motes as shown in Figure 7.

5.2.1 MAC Performance with Different DOI. In this section, we set VDOI as 0 , to evaluate the performance of MAC layer with different DOI values. In the next section, we investigate the performance sensitivity to DOI variance, by setting 
VDOI greater than 0 .

In the initial setup, we use Geographic Forwarding (GF) in the routing layer and compare the MAC performance between 802.11 and CSMA. We found that the MAC loss ratio increases rapidly with the increase of DOI values (Figure 12(a)). However, 802.11 and CSMA yield roughly the same results. We realize that MAC performance can be strongly affected by routing, because an incorrect routing decision might lead to the failure at MAC layer. For instance, the routing layer designates that the MAC layer send a packet to a node that is out of reach. So we repeat the experiments with the AODV protocol as the routing layer. We find that the MAC loss ratio increases slightly with the increase of DOI values. Such a discrepancy is a strong indication that the radio irregularity has a much larger impact on routing protocols than MAC protocols. We explain this in more detail in Section 6.

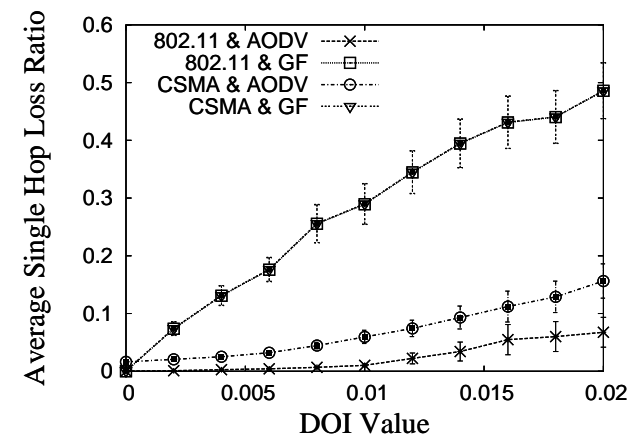

(a) Loss Ratio vs. DOI

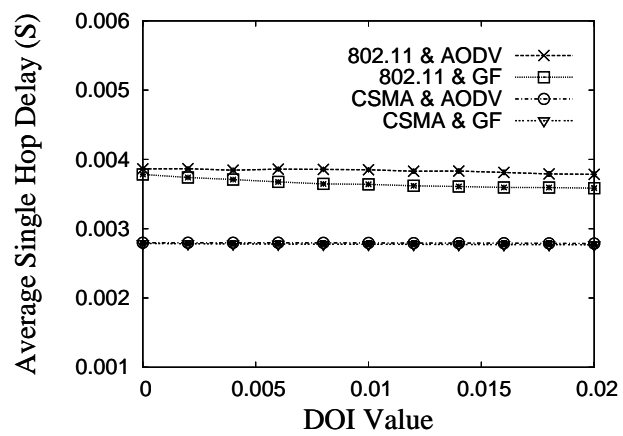

(b) Average Single Hop Delay vs. DOI

Fig. 12. MAC Performance with Different DOI Values

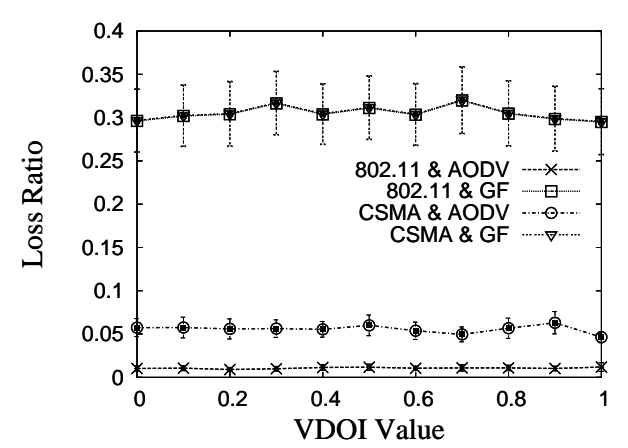

(a) Loss Ratio vs. VDOI

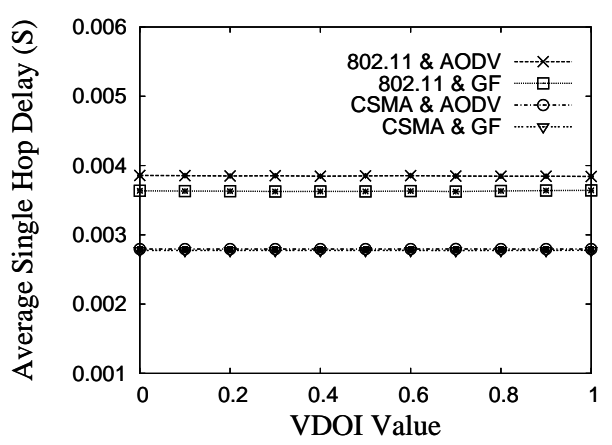

(b) Average Single Hop Delay vs. VDOI

Fig. 13. MAC Performance Sensitivity to Different VDOI Values

From Figure 12(b), we can see that with the increase of DOI values, the average single hop delay remains almost the same. The reason is that increasing the DOI 
value only increases the communication asymmetry, but not the congestion. This is also a confirmation that packet loss in Figure 12(a) is not due to congestion.

5.2.2 Performance Sensitivity to Different VDOI. This section is to explore whether DOI variance in a system has impact on the MAC performance. In the simulation, we set DOI as 0.01 and vary VDOI from 0 to 1 , in steps of 0.1 , and present the simulation results in Figure 13.

From Figure 13, we observe that when VDOI varies from 0 to 1 , neither the average single hop loss ratio nor the average single hop delay varies much. The possible reason is that, statistically, while one portion of nodes have larger DOI values and hence more irregular radio, another portion of nodes will have smaller DOI values and hence less irregular radio. So their effects are cancelled by each other, and the system-wide MAC performance is not sensitive to different VDOI values.

5.2.3 MAC Performance with Different VSP. In this experiment, we set the DOI value to 0 , which means that the radio range is isotropic. However, different VSP values make radio ranges different among nodes.

The results shown in Figure 14 are similar to the results shown in Figure 12, which we obtain by varying the DOI values. The average single hop delay remains almost the same, because the different sending powers only increase the degree of communication asymmetry, but not the congestion.

The loss ratio increases with the increase of VSP values because the irregularity results in more asymmetric links. The loss ratio when AODV is used is much lower than that when GF is used, because asymmetric links have a larger impact on GF than on AODV. This result indicates that varying the VSP values has a much larger impact on routing protocols than on MAC protocols, which is similar to the behavior we observed by varying the DOI values.

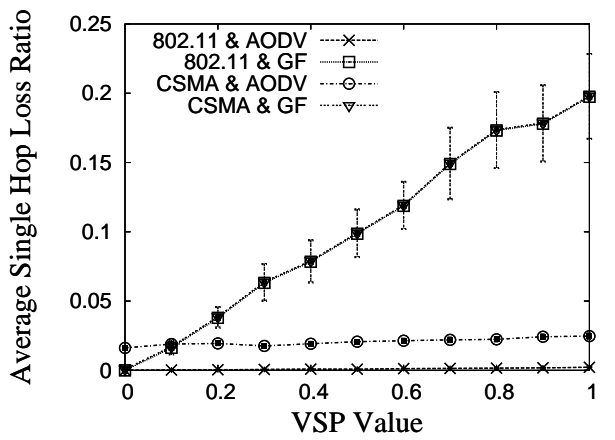

(a) Loss Ratio vs. VSP

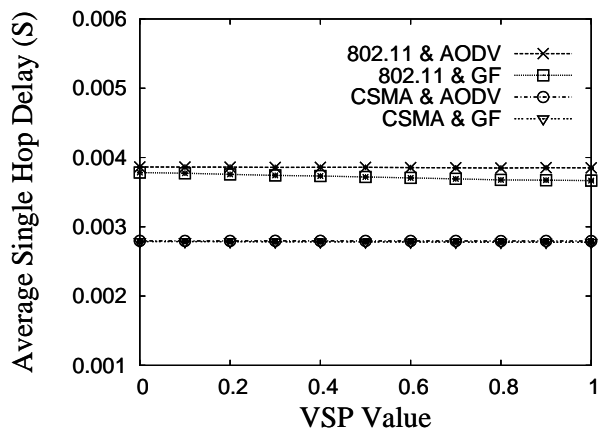

(b) Average Single Hop Delay vs. VSP

Fig. 14. MAC Performance with Different VSP Values

\section{IMPACT ON ROUTING LAYER}

In this section, we analyze and quantify the impact of radio irregularity on routing protocols. We first discuss three techniques that are widely used in most routing 
protocols: path-reversal, multi-round discovery, and neighbor discovery. Our analysis shows that both path-reversal and neighbor-discovery are greatly influenced by radio irregularity. However, the multi-round discovery technique is able to deal with radio irregularity, but with relatively high overhead. Our simulation results also show that radio irregularity has a great impact on Geographic Forwarding (GF), but a small impact on AODV and DSR.

\subsection{Logical Analysis of the Impact}

In this section, we study the influence of radio irregularity on path-reversal, multiround discovery, and neighbor-discovery techniques. We also quantify this influence in two cases. In one case, path loss difference is the main reason of radio irregularity and link asymmetry, and in the second case, difference in radio sending power is the main reason.

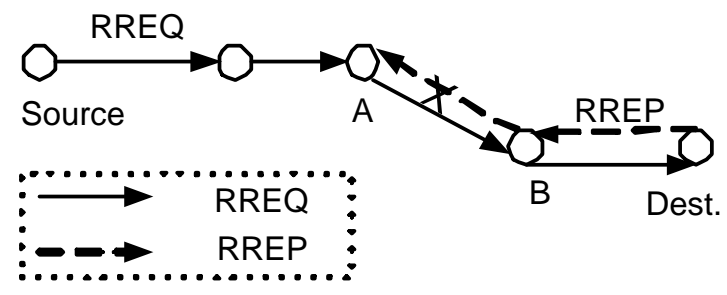

Fig. 15. Impact on Path-Reversal Technique

6.1.1 Impact on Path-Reversal Technique. Protocols that use path-reversal technique are built based on the assumption that if there is a path from node $\mathrm{A}$ to node $\mathrm{B}$, there is also a reverse path from node $\mathrm{B}$ to node $\mathrm{A}$. The path may consist of a single link or multiple links. Most on-demand routing protocols used in ad hoc networks such as AODV [Perkins and Royer 1999], DSR [Johnson and Maltz 1996], Direct Diffusion [Intanagonwiwat et al. 2000] and LAR [Ko and Vaidya 1998] depend on this technique.

Radio irregularity may result in asymmetric links and hence, it may have an adverse impact on protocols that use path-reversal techniques. For example, in Figure 15, node B can hear node A, but node A cannot hear node B. So even though there is a path from source $\mathrm{S}$ to destination $\mathrm{D}$, we cannot assume that the reverse path from $\mathrm{D}$ to $\mathrm{S}$ exists. So during route discovery, if source $\mathrm{S}$ broadcasts a route request (RREQ) to discover the path to destination $\mathrm{D}$, it may not be possible to deliver the reply (RREP) message to source $\mathrm{S}$ along the reverse path, even though node $\mathrm{D}$ replies to the request. In such a case, the route discovery fails.

The above analysis leads one to believe that it would be inappropriate to use any routing protocol that uses path-reversal in route discovery, such as AODV, DSR, DD and LAR, in an asymmetric environment, because they would have a very high loss ratio. However, the simulation results we present later show that AODV and DSR work reasonably well despite the asymmetric nature of communication. The 
reason is that in addition to path-reversal technique, these routing protocols also use the multi-round discovery, which is capable of dealing with asymmetry, but with a high overhead.

6.1.2 Multi-Round Discovery Technique. In AODV and DSR, the RREQ is broadcast towards the destination $D$. So node $D$ receives RREQ messages from multiple paths, as shown in Figure 16. It chooses one of the many available paths to send the RREP message back to source S, according to some runtime configurable parameter, such as the RREQ arrival time, path load, or end-to-end delay of the path. If the reverse path does not exist, the RREP fails to arrive at sender $\mathrm{S}$ and the route discovery is repeated due to timeout. In the next attempt, thanks to the random nature of flooding, node $\mathrm{D}$ might receive a RREQ message from another path, which happens to be a symmetric connection.

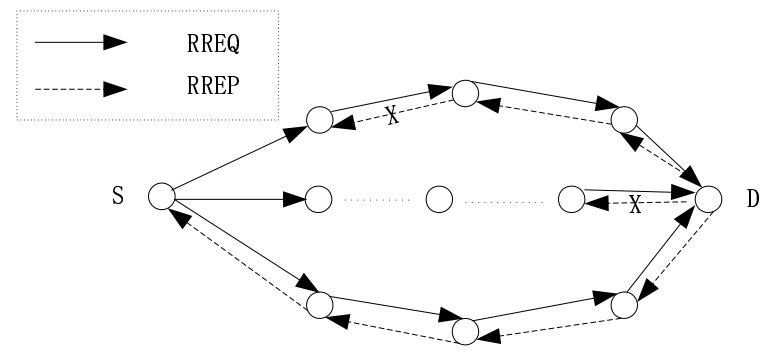

Fig. 16. Route Discovery Using Rediscovery Technique

The chance to establish a symmetric connection increases after retries. If there is no limitation on the number of retries, a symmetric path will sooner or later be discovered on the condition that such a path exists. We note that the rediscover technique provides a viable way to work around the effects of asymmetry, but with significant overhead. Also the path reinforcement scheme presented in [Intanagonwiwat et al. 2000] can reduce the impact of path-reversal technique, by continuously monitoring performances in multiple paths and reinforcing the best path.

6.1.3 Impact on Neighbor Discovery Technique. Many location-based routing protocols [He et al. 2003][Karp and Kung 2000][Karp 2000] use the neighbor discovery technique in order to maintain the neighborhood information. However, the neighbor discovery technique works well only if the links are symmetric. For example, in Figure 17, node A discovers its neighbors by receiving beacons. Node A might choose one of its neighbors, node B, C, or D for forwarding packets. However, if node $\mathrm{A}$ picks node $\mathrm{B}$ which is unable to hear node $\mathrm{A}$, node $\mathrm{B}$ will never receive the packet forwarded by node $\mathrm{A}$. If node $\mathrm{A}$ does not retry its transmission with the other neighbors, the transmission of the packet will fail. So the routing protocol based on the neighbor discovery technique is subject to failures when communication is asymmetric. 


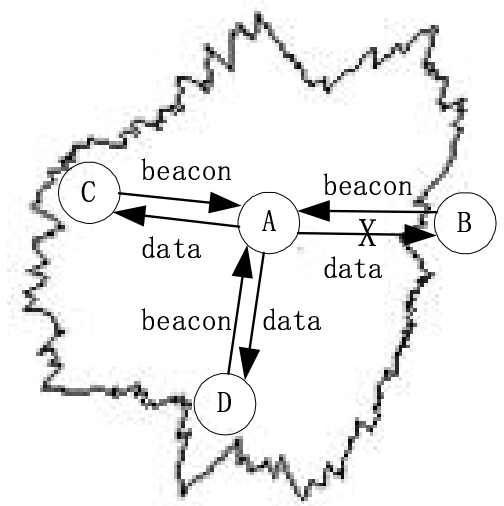

Fig. 17. Impact on Neighbor Table Technique

\subsection{Quantitative Analysis of the Impact}

In this section, we quantify the performance penalty of radio irregularity, through four sets of experiments. In each set, we measure four metrics: end-to-end (E2E) loss ratio, average E2E delay, number of control packets, and energy consumption.

We first measure the routing performance with different DOI values, and then investigate the performance sensitivity to different VDOI values. In the third set of experiments, different VSP values are configured to explore the routing performance. In the fourth set of experiments, we take the GF routing protocol as an example, to investigate the performance changes when combinations of different DOI and VSP values are used.

Before we analyze the performance evaluation, we'd like to explain how GF [Karp 2000] works. In GF, each node beacons its ID and location periodically, so that each node can maintain up to date neighbor information. When the application requests to send a packet to a specified destination, GF compares the distance of each neighbor to the destination, and forward the data packet to the neighbor that is closest to to the destination. This routing strategy is repeated by all intermediate nodes that participate in packet forwarding, until the data packet is finally received by the destination.

6.2.1 Routing Performance with Different DOI. In this section, we set VDOI to 0 to evaluate routing performance with different DOI values. In the following section, we set VDOI greater than 0 to investigate routing performance sensitivity to $\mathrm{DOI}$ variance.

Figure 18(a) shows that GF is greatly influenced by radio irregularity. It loses $84.5 \%$ packets when the DOI is 0.02 . The reason is that according to the greedy forwarding rule, GF tends to choose a node near the border, which is more likely to have an asymmetric link with the sender. AODV and DSR perform well because they use multi-round discovery, exploring alterative paths to find a symmetric connection. However, they achieve a low loss ratio at the cost of increased overhead in control packets shown in Figure 18(c).

In Figure 18(b), the average E2E delay of DSR and AODV increases with the increase of DOI values. That is because more rounds of route discovery are needed 


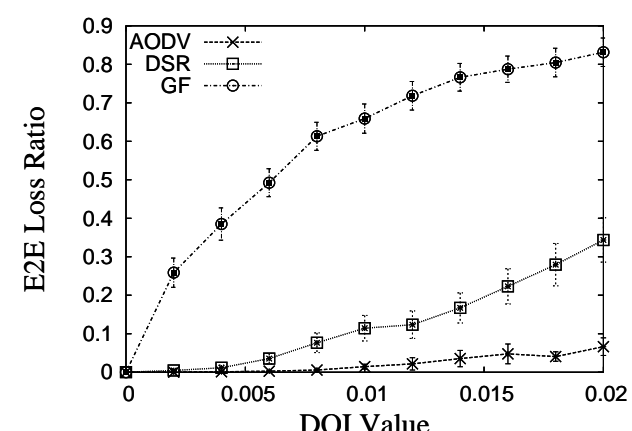

(a) E2E Loss Ratio vs. DOI

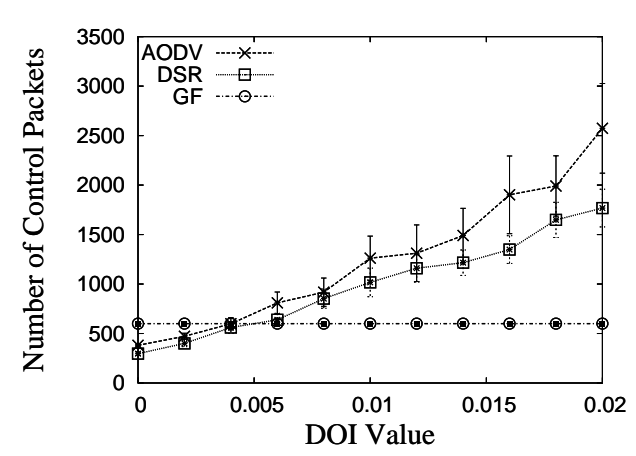

(c) Number of Control Packets vs. DOI

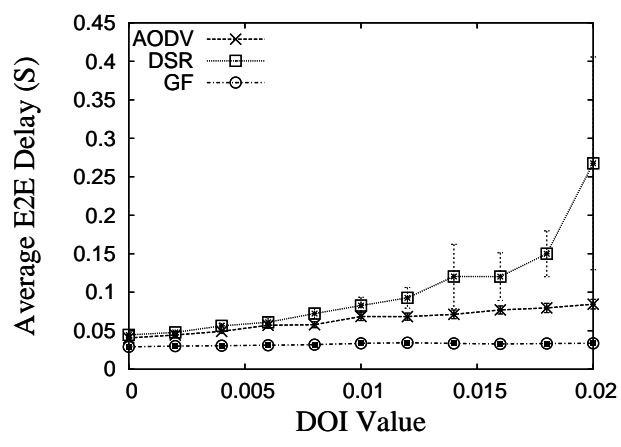

(b) Average E2E Delay vs. DOI

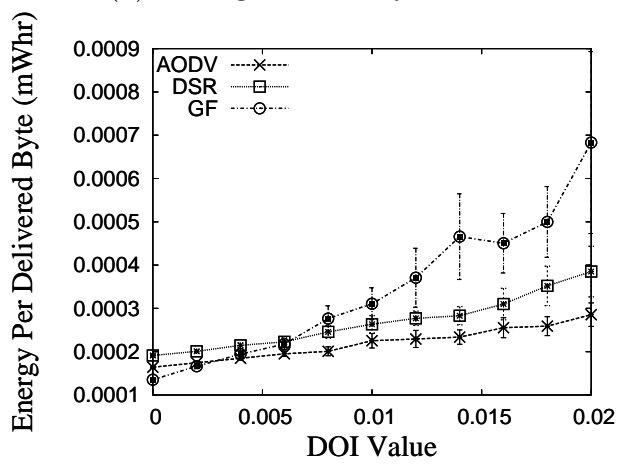

(d) Energy Consumption vs. DOI

Fig. 18. Routing Performance with Different DOI Values

as the radio irregularity increases. In Figure 18(b) DSR has a higher delay than AODV, because the source routing technique in DSR adds the whole path in the header of data packets, which increases the transmission time. However, the E2E delay of GF remains the same because packets in GF either go through successfully or get dropped.

Figure 18(c) shows that while AODV and DSR need more control packets to do multi-round discovery, when the DOI value increases GF needs only a constant number of control packets for neighbor exchange.

Figure 18(d) presents the energy consumption normalized according to useful work completed. It is measured as the energy consumed for each successfully delivered end-to-end data byte. Figure 18(d) informs that AODV, DSR and GF all consume more energy to deliver a useful data byte through multiple hops, when the DOI value increases. This is because the increased radio irregularity leads to increased asymmetry links, which result in increased retransmission to deliver the same amount of useful data. Moreover, as shown in Figure 18(a), GF delivers less useful data than AODV and DSR, and DSR delivers less useful data than AODV, with the increase of DOI values. Accordingly, among the three routing protocols, GF is less energy efficient than AODV and DSR, and DSR is less energy efficient than AODV, as shown in Figure 18(d). 
6.2.2 Performance Sensitivity to Different VDOI. This section is to analyze whether the DOI variance in a system has impact on routing performance. In the simulation, we set DOI as 0.01 and vary VDOI from 0 to 1 , in steps of 0.1 .

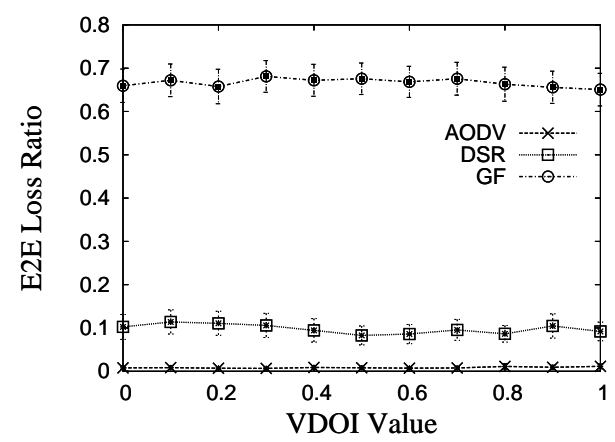

(a) E2E Loss Ratio vs. VDOI

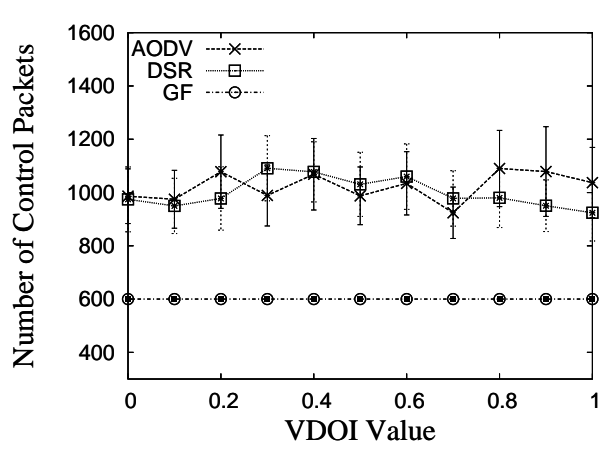

(c) Number of Control Packets vs. VDOI

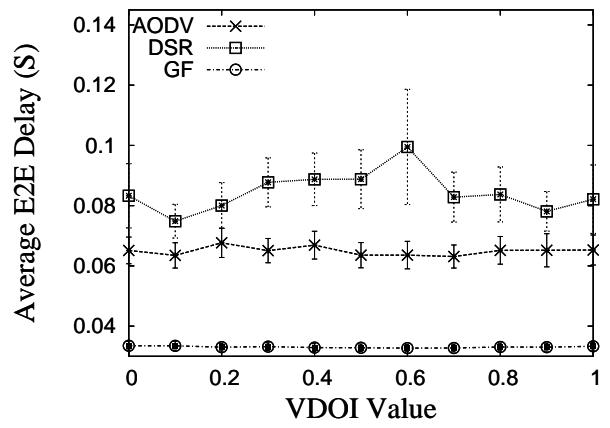

(b) Average E2E Delay vs. VDOI

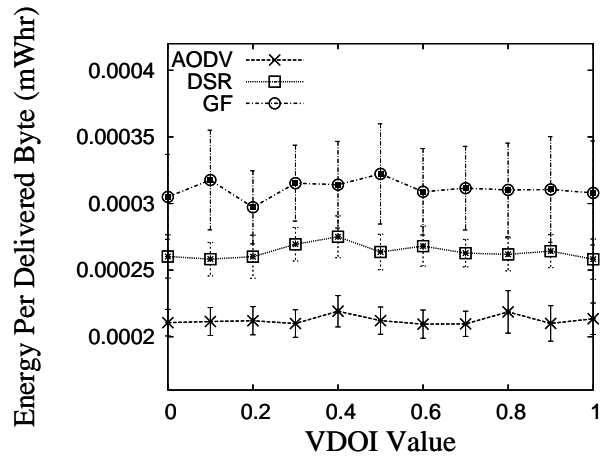

(d) Energy Consumption vs. VDOI

Fig. 19. Routing Performance Sensitivity to Different VDOI Values

From Figure 19, we observe that when the VDOI value varies from 0 to 1 , none of the four metrics, E2E loss ratio, average E2E delay, number of control packets and energy consumption per delivered data byte, shows clear performance variance statistically. This is because on one hand, some nodes get larger DOI values and have more irregular radio patterns, on the other hand, some other nodes get smaller DOI values and have less irregular radio signals. They cancel their effects with each other, and we observe no significant performance changes with different VDOI values.

6.2.3 Routing Performance with Different VSP. In Figure 20, the impact of radio irregularity on the routing layer is measured for different DOI values. In this section, we measure the impact of radio irregularity on the routing layer by varying the VSP values. From our results, we find that an increase of the VSP value has a similar impact on AODV, DSR and GF, as an increase of the DOI value, because both lead to a higher degree of irregularity and therefore, a higher degree of link asymmetry. 
From Figure 20(a), we see that all routing protocols have higher loss ratios when the VSP value is increased, because there are more asymmetric links. GF has a much higher loss ratio than that of AODV and DSR, because GF uses neighbor discovery and tends to choose the same node near the border of the radio range as the candidate, while AODV and DSR use multi-round discovery to try different paths.

As in the case of larger DOI values, larger VSP values result in more asymmetric links, which lead to larger average E2E delays (Figure 20(b)) and higher energy consumption per delivered data byte(Figure 20(d)). However, GF does not require more beacons, so there is no increase in the control packets (Figure 20(c)) and the delay remains the same (Figure 20(a)). The energy consumption of GF for each delivered data byte increases sharply with the increase of VSP values, because its packet loss increases quickly.

To summarize, as DOI and VSP increase, radio irregularity has a greater adverse impact on the GF protocol compared to on-demand routing protocols that use multi-round discovery such as AODV and DSR.

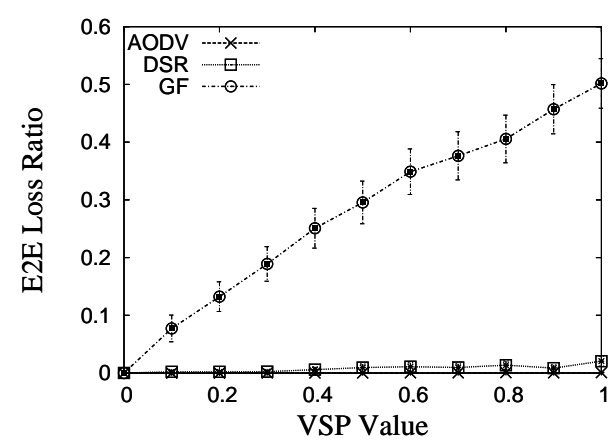

(a) E2E Loss Ratio vs. VSP

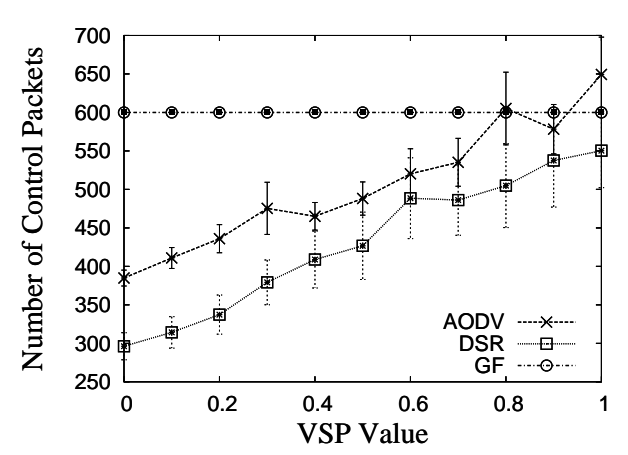

(c) Number of Control Packets vs. VSP

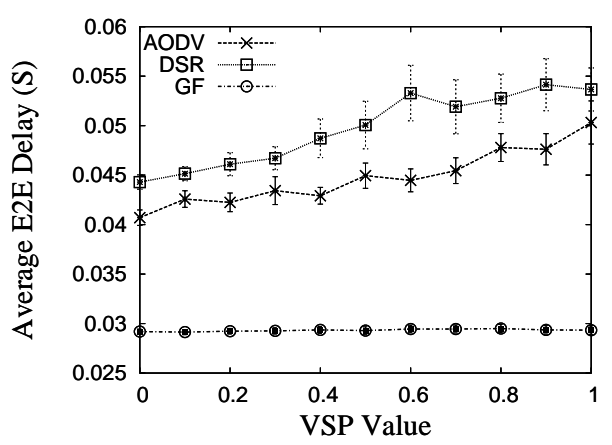

(b) Average E2E Delay vs. VSP

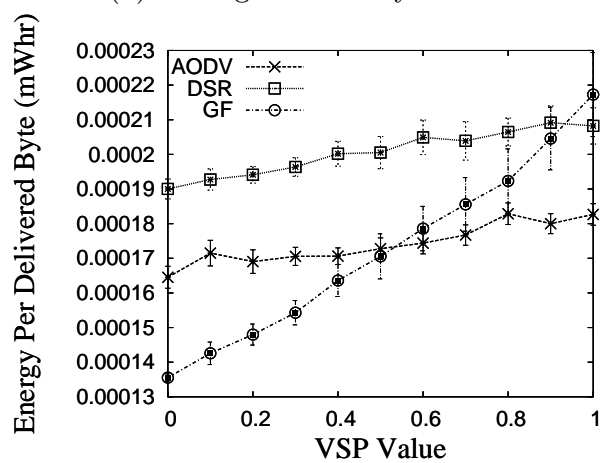

(d) Energy Consumption vs. VSP

Fig. 20. Routing Performance with Different VSP Values

6.2.4 Routing Performance with Different DOI-VSP Combinations. In Section 6.2.1 and 6.2.3, we evaluate the impact of radio anisotropy and heterogeneous 
sending powers on routing performance, by setting different DOI and VSP values separately. In this section, we explore their composite impact on the routing protocols. We take the GF routing protocol as an example, and use combinations of different DOI and VSP values to evaluate GF's packet loss ratio.

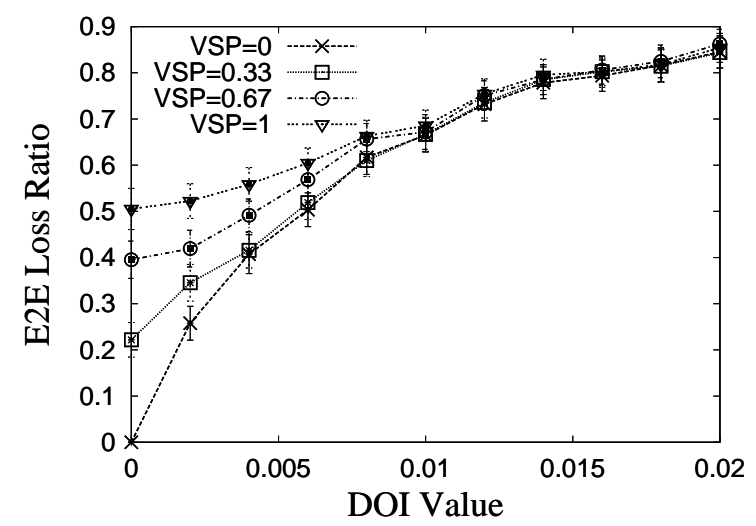

Fig. 21. GF Performance with Different DOI-VSP Combinations

As Figure 21 illustrates, for all the configured VSP values, whether it is 0 or greater than 0, a larger DOI value always leads to a larger E2E loss ratio. The routing performance decreases similarly as what we observe in Figure 18(a) when the VSP value is set to 0 . On the other hand, for all the DOI values we use, a larger VSP value also leads to a larger E2E loss ratio, which is similar to what we observe in Figure 20(a) when the DOI value is set to 0. This shows that the impact of DOI and VSP do not cancel each other.

However, the impact of DOI and VSP on routing performance do not arithmetically accumulate, according to the results in Figure 21. This reflects that the asymmetric channels caused by DOI overlap with those caused by VSP in some locations of the system. The more the asymmetric channels overlap, the larger the gap between the composite E2E loss ratio and the sum of $\mathrm{E} 2 \mathrm{E}$ loss ratios when either of them is set to 0 . This is why the distances among the four curves shown in Figure 21 get closer while the DOI value increases.

\section{IMPACT ON LOCALIZATION}

In this section, we explore the impact of radio irregularity on localization protocols. We do not try to cover every localization protocol in detail, since that is beyond this paper's discussion. We take some popular techniques and protocols from the localization family and analyze the impact. We also present performance evaluation of the Centroid protocol, as an example of quantitative analysis.

Radio irregularity has a great impact on the localization protocols that use the Received Signal Strength Indicator (RSSI) technique, such as RADAR [Bahl and Padmanabhan 2000] and SpotOn [Hightower et al. 2000]. The RSSI technique assumes that once the distance between the transmitter and receiver is determined, the RSSI value is determined, and vice versa. However, in our experiments with 
Table II. Simulation Configuration Two

\begin{tabular}{||l|l||}
\hline TERRAIN & $(150 \mathrm{~m}$ X 150m) \\
\hline Node Number & 400 (including anchors) \\
\hline Node \& Anchor Placement & Random \\
\hline Routing Protocol & GF \\
\hline MAC Protocol & 802.11 (DCF) \\
\hline Radio Layer & RADIO-ACCNOISE \\
\hline Radio Model & RIM \\
\hline Nominal Radio Range & $65 \mathrm{M}$ \\
\hline Radio Bandwidth & $200 \mathrm{~Kb} / \mathrm{s}$ \\
\hline
\end{tabular}

MICA2 motes (Figure 2), the RSSI value varies when the receiver is put at different propagation directions from the transmitter, even though the distance between them is invariant, 10 feet in Figure 2(a) and 20 feet in Figure 2(b). Accordingly, the RSSI technique is misleading in calculating locations when the radio propagation direction is disregarded.

In DV-HOP [Niculescu and Nath 2003], the anchor nodes flood their locations throughout the whole network. Any node receiving this message records its hopcount to corresponding anchors. Then with these hop-counts to anchors, with the average distance per hop and with the anchors' locations, each node can figure out its own location. However, the radio range is not isotropic, and the communication ranges do not have an invariant value in different propagation directions. So the distance of each hop varies greatly, depending on the degree of radio irregularity. So it is misleading to calculate a node's distance to an anchor as the product of the hop-count and the average distance per hop.

Radio irregularity has a great impact on the Centroid algorithm. In Centroid, a node's location is calculated as the geographic center of all anchors it hears. This idea does not work well because a node that can hear $N$ anchors is not necessarily located exactly at the geographic center of the $N$ anchors. When we consider the fact of irregular radio, the performance becomes worse, which can be observed in the simulation result illustrated in Figure 22. As before, the simulation is conducted in GloMoSim, and the simulation configuration is given in Table II.

From Figure 22(a), we observe that with the increase of DOI values, the localization error keeps increasing, with all the settings of the average Anchor Heard $(\mathrm{AH})$, which is defined as the average number of anchors heard by a node and used during location estimation. For example, when DOI is 0 and AH is 20, the radio is spherical and the localization error is $33.7 \%$ of the nominal radio range. But when the DOI increases to 0.02 and $\mathrm{AH}$ remains 20, the radio becomes very irregular and the localization error increases to $54.7 \%$ of the nominal radio range. The decreased Centroid performance is caused by the increased radio irregularity, since larger DOI values lead to more anisotropic radio patterns.

Similar experiments are repeated with different VSP values(Figure 22(b)), and we find that Centroid's localization error also increases with increasing VSP.

\section{IMPACT ON TOPOLOGY CONTROL}

In this section, we take GAF [Xu et al. 2001], a typical topology control protocol, as an example to study the impact of radio irregularity. In GAF, the deployment 


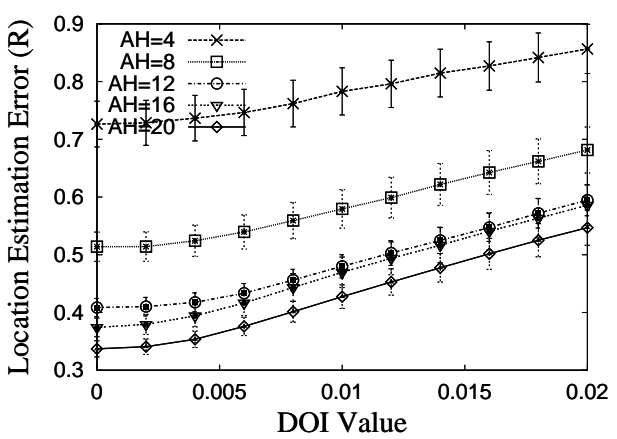

(a) Performance with Different DOI Values

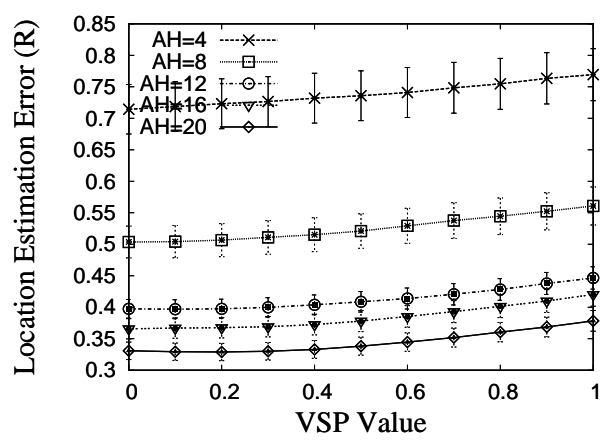

(b) Performance with Different VSP Values

Fig. 22. Centroid's Performance with Different Radio Irreguarity

terrain is divided into virtual grids. In each grid, one node is chosen to stay awake and the others are put to sleep to save power. But at any time, the communication connectivity among adjacent grids must be maintained. In order to maintain the connectivity, the radio communication range $R$ and the grid side length $r$ must satisfy the following relations:

$$
r \leq \frac{R}{\sqrt{5}}
$$

Since radio is in fact irregular and the communication range is not spherical, it is hard to determine the value of the parameter $R$. In GAF, $R$ is defined as the nominal radio communication range. However, using the nominal radio range $R$ makes it impossible to guarantee the communication connectivity among adjacent grids, in the presence of anisotropic radio.

In order to investigate GAF's communication connectivity in the case of radio irregularity, we implement GAF in GloMoSim and present the simulation results in Figures 23 and 24. In the simulation, we use the configuration setting presented in Table II.

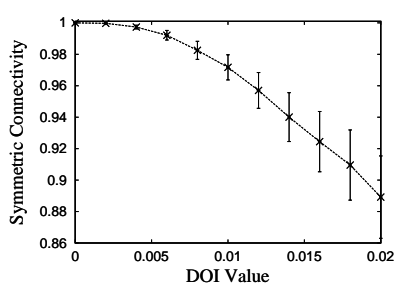

(a) Symmetric Connectivity

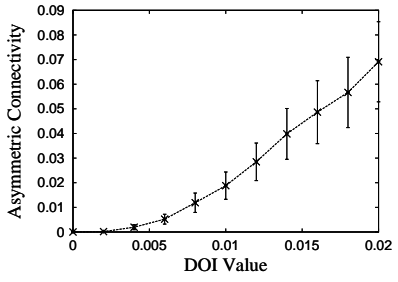

(b) Asymmetric Connectivity

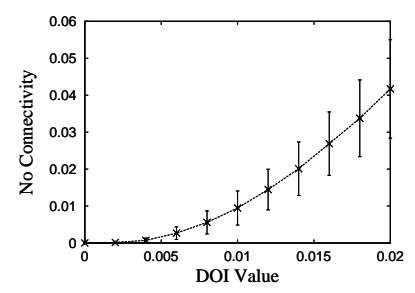

(c) No Connectivity

Fig. 23. GAF's Connectivity Status with Different DOI Values

We measure the connectivity status among adjacent grids with different DOI and VSP values. From Figure 23, we observe that the percentage of symmetric connectivity decreases with the increase of DOI values. When the radio range is 


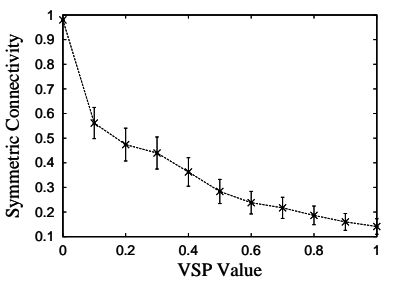

(a) Symmetric Connectivity

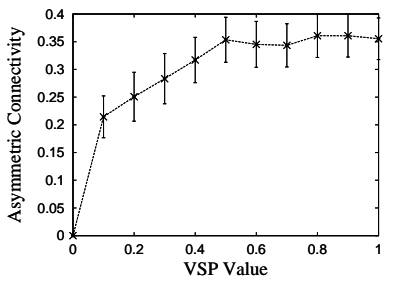

(b) Asymmetric Connectivity

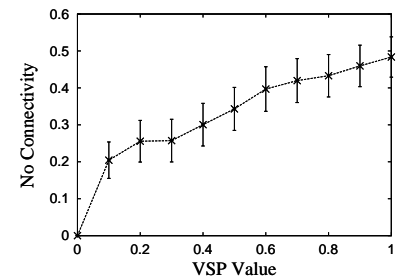

(c) No Connectivity

Fig. 24. GAF's Connectivity Status with Different VSP Values

spherical, i.e., DOI is 0, all connections are symmetric. But when DOI increases to 0.02 and the radio becomes irregular, the percentage of symmetric connections decreases to $89 \%$ (Figure 23(a)), and $7 \%$ of the connections become asymmetric (Figure 23(b)) and $4 \%$ of the connections totally get broken (Figure 23(c)). This is because of the radio irregularity. When the radio range becomes more and more anisotropic, the original symmetric connectivity becomes asymmetric, and the original asymmetric connections are broken in both directions.

We repeat the experiments with different VSP values, and similar results are observed in Figure 24. In Figure 24, when all nodes have the same sending power and the system is homogeneous, i.e., VSP is 0, all connections are symmetric. But when the VSP value increases to 1 , only $15 \%$ of the connections are symmetric (Figure 24(a)), and there are 36\% asymmetric connections (Figure 24(b)) and $49 \%$ connections are completely broken (Figure 24(c)). The reason for the decreased communication connectivity is the increasing heterogeneity in devices' sending powers, which results in greater difference in nominal radio ranges among different devices and leads to worse communication connectivity.

\section{SOLUTIONS FOR RADIO IRREGULARITY}

Having analyzed the causes and impact of radio irregularity, the key results can be summarized as follows:

- Radio irregularity is a common and non-negligible phenomenon in wireless systems. Link asymmetry is an upper layer phenomenon produced by irregular radio signals in the radio layer. And asymmetry links directly lead to MAC and routing failures.

- Radio irregularity has a greater impact on the routing layer than MAC layer.

- Routing protocols, such as AODV and DSR, that use multi-round discovery technique, can deal with radio irregularity, but with a high overhead.

- Routing protocols, such as geographic forwarding, which are based on neighbor discovery technique, are severely affected by radio irregularity.

- Radio irregularity results in larger localization errors and makes it harder to maintain communication connectivity.

Based on both analytical and experimental results, we present eight potential solutions to improve the protocol performance in the presence of radio irregularity. 
We first describe the Symmetric Geographic Forwarding, the Asymmetry Detection Service and the Bounded Distance Forwarding solutions in detail and discuss their performance evaluation. We then follow that by briefly describing five other solutions.

\subsection{Symmetric Geographic Forwarding}

In location-based protocols, such as GF and GPSR, the beacon message only contains the node's ID and position. In our new Symmetric Geographic Forwarding (SGF) solution, we allow a node to add the IDs of all its neighbors it has discovered into the beacon message. When a node receives a beacon message, it registers the sender as its neighbor in its local neighbor table, and then checks whether its own ID is in the beacon message. If the receiver finds its own ID in the neighbor list in the beacon message, then it marks the communication link connecting it to the sender as SYMMETRIC. Otherwise, it marks the communication link between them as ASYMMETRIC. Whenever a node needs to forward a packet, it selects only those neighboring nodes with which it is connected through SYMMETRIC links. Here we must emphasize that when a node broadcasts a beacon message, it should add the IDs of the nodes with which it has SYMMETRIC connectivity as well as those nodes with which it has ASYMMETRIC connectivity.

We simulate SGF in GloMoSim. We find that SGF maintains most of the advantages of GF, such as scalability, and the absence of flooding. Furthermore, SGF is able to deal with asymmetry as effectively as the multi-path route discovery protocols, such as AODV and DSR, but at lower cost. The simulation setup use the same configuration as mentioned in Table I.

9.1.1 SGF Performance with Different DOI. In this experiment, we incrementally increase the degree of irregularity (DOI) to measure the SGF performance.

From Figure 25(a), we observe that SGF has a significantly lower loss ratio than that of GF, and performs as well as AODV. This is because it avoids forwarding data along asymmetric links. From Figure 25(b), we observe that SGF has almost the same average E2E delay as that of GF. The delay is much lower than that of ADOV and DSR. An interesting point from Figure 25(c) is that SGF consumes the same number of control packets as that of GF, and the number of control packets remains the same with the increase of DOI values. From Figure 25(d), it is observed that GF has a rapidly increased energy consumption for each successfully delivered data byte, with the increase of DOI values. But AODV, DSR and GF have comparatively slow increases of energy consumption for each delivered data byte. This is because radio irregularity has a greater impact on GF than on AODV, DSR and SGF, and GF suffers the most packet loss. DSR is less energy efficient than AODV and SGF, because it has more packet loss (Figure 25(a)) as well as more control overhead (Figure 25(c)) than AODV and SGF. On the other hand, SGF exhibits the least increased energy consumption among these four routing protocols, because its packet loss is as low as that of AODV (Figure 25(a)), and its control overhead is as low as that of GF (Figure 25(c)).

9.1.2 SGF Performance with Different VSP. Similar conclusions can be drawn from the results in Figure 26. Compared with GF, SGF has a much lower loss ratio, 


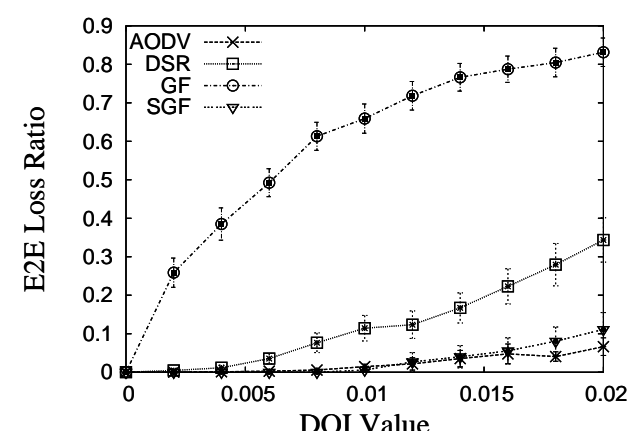

(a) E2E Loss Ratio vs. DOI

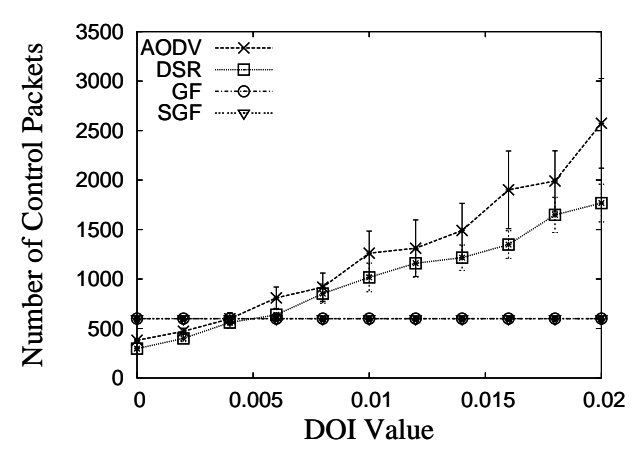

(c) Number of Control Packets vs. DOI

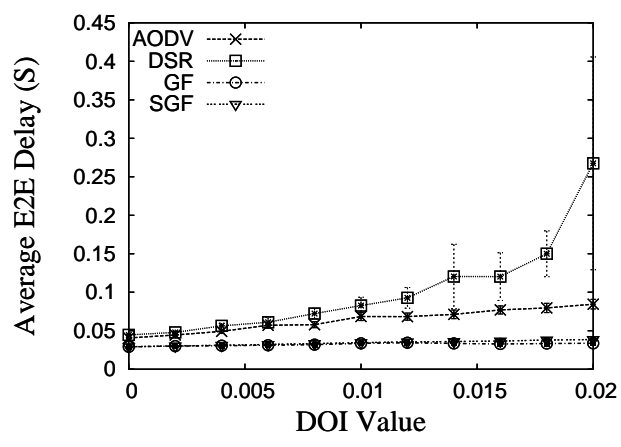

(b) Average E2E Delay vs. DOI

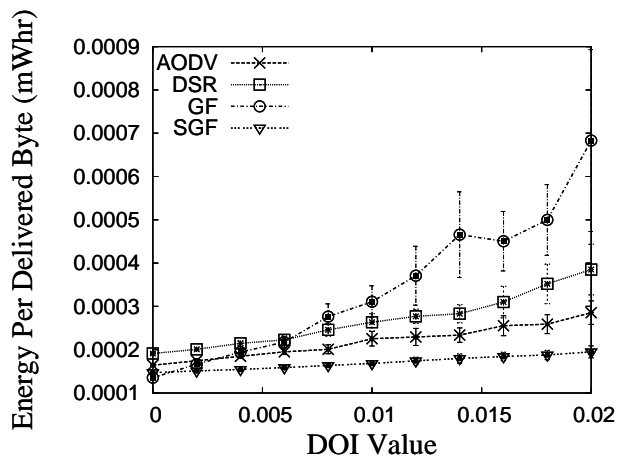

(d) Energy Consumption vs. DOI

Fig. 25. SGF Performances with Different DOI Values

almost the same average E2E delay, and the same number of control packets, with an increase of the VSP value. The loss ratio of SGF is comparable to that of AODV and DSR. However, SGF has a much lower average E2E delay, a constant number of control packets, and a much lower energy consumption for each delivered data byte. SGF consumes almost constant energy with an increase of the VSP value. In contrast, GF suffers a sharply increased energy consumption for each delivered data byte, because of its rapidly increased packet loss. Plus, AODV and DSR also consume more energy for each delivered data byte compared to SGF, on account of two reasons. First, AODV and DSR need more control overhead than SGF, as shown in Figure 26(c). Second, AODV has the same level of packet loss ratio as that of SGF while DSR drops more useful data packets than SGF (Figure 26(a)).

To summarize, the SGF protocol not only maintains GF's scalability, but also successfully deals with radio irregularity. Compared with AODV and DSR, it achieves similar delivery ratio in the presence of radio irregularity with a lower E2E delay, a lower number of control packets and lower energy consumption.

\subsection{Asymmetry Detection Service}

The SGF provides a basic prototype of incorporating symmetric detection into routing protocols. In a running system, more sophisticated algorithms should be 


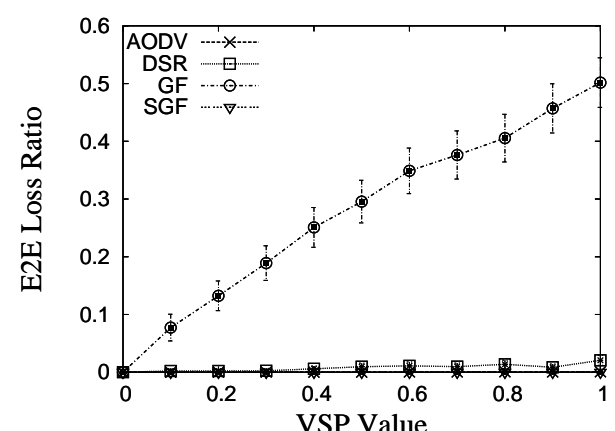

(a) E2E Loss Ratio vs. VSP

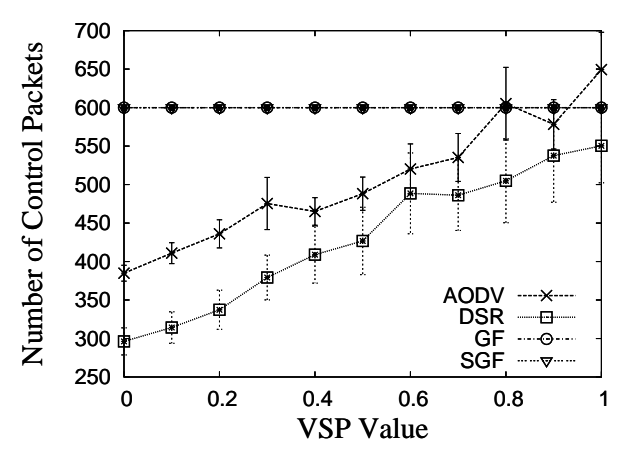

(c) Number of Control Packets vs. VSP

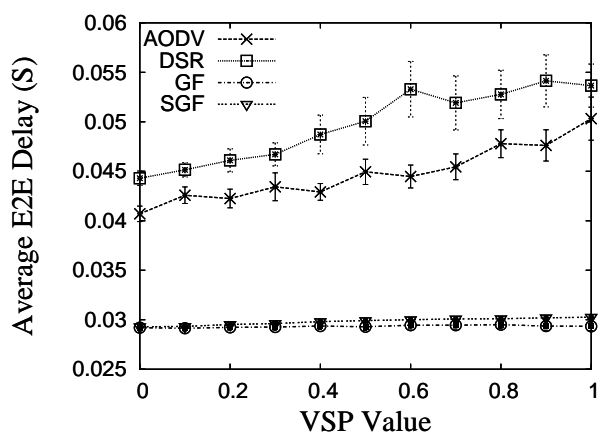

(b) Average E2E Delay vs. VSP

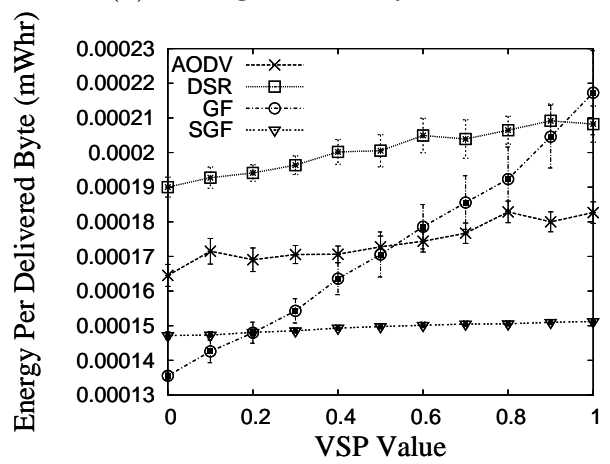

(d) Energy Consumption vs. VSP

Fig. 26. SGF Performance with Different VSP

introduced to deal with engineering issues ${ }^{6}$. In this section, we implement a general Asymmetry Detection Service in the VigilNet System [He et al. 2004] developed by University of Virginia.

In the Asymmetry Detection Service, the same idea is used to mark a link as SYMMETRIC or ASYMMETRIC as what is used in SGF. However, to deal with engineering issues in running systems, this marking process is repeated several times to get a statistical evaluation of a link's symmetric communication quality. Only those links that have higher symmetric communication qualities than the specified threshold are available for upper layers, and all other links are blocked from higher layer protocols.

In the VigilNet System, a communication backbone is built to relay messages back to the base station. The communication backbone is established using a classic spanning tree algorithm [Cormen et al. 2002], with the base station as the spanning tree's root. During the construction of the spanning tree, the Asymmetry Detection

\footnotetext{
${ }^{6}$ Engineering issues mean the system dynamics caused by the unpredictable system deployment environment, such as the changing temperature and humidity levels, the swinging trees and the jumping bugs, as well as the system dynamics caused by passing cars and human beings walking around. All these engineering issues bring communication dynamics into running system experiments.
} 
Service is called and only symmetric links are used. We measure the performance evaluation of the Asymmetry Detection Service, by counting the percentage of nodes that are able to report back their status information successfully through the communication backbone. We conduct the experiment with 27 MICA2 devices and the result is given in Figure 27.

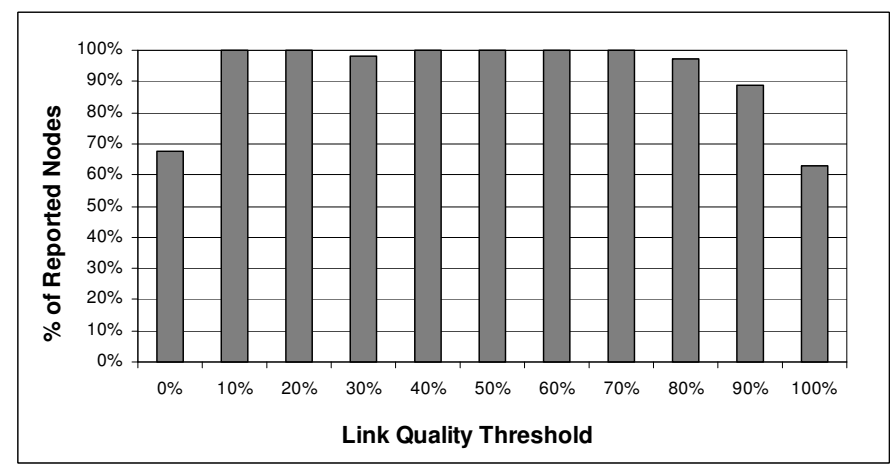

Fig. 27. Performance Evaluation of Asymmetry Detection Service

When the Asymmetry Detection Service is disabled, by setting the link quality threshold to 0 as shown in Figure 27, only $67.4 \%$ nodes are able to successfully report information back to the base station, because the communication backbone consists of a large portion of asymmetric links, and the data packets can not be correctly relayed back to the base station following the reversed path from the spanning tree's leaves to its root, the base station. However, when the Asymmetry Detection Service is used, we observe that almost all nodes are able to successfully report back to the base station. The spanning tree backbone works well when the link quality threshold is set from $10 \%$ to $70 \%$. This performance improvement is caused by the Asymmetry Detection Service, which cuts off unidirectional links, and contributes to establishing the reliable communication backbone.

We are aware that the system still performs very well, even when the link quality threshold is set very low, as low as $10 \%$. This is because MAC layer retransmission is used, in case of communication failures. However, the MAC layer retransmission alone can not achieve this good performance. When we disable the Asymmetry Detection Service by setting the link quality threshold to 0 and only use the MAC layer retransmission, the system performance is very poor, only $67.4 \%$ of the nodes report back to the base station.

On the other hand, when the link quality threshold keeps increasing and is close to $100 \%$, the system performance decreases. This is because when Asymmetry Detection Service blocks all links that do not have $100 \%$ link qualities, there are not enough links available to build the communication backbone, and network partition happens.

The scheme we proposed here is related to approaches proposed in [Woo et al. 2003], in which each node snoops on the channel and eavesdrops on communications over time, to evaluate the inbound channel quality. Since routing is based 
on outbound (transmission) links, a separate phase is used in [Woo et al. 2003] for nodes to exchange inbound quality information to build up outbound quality information. However, our scheme is different. In our scheme, each node beacons periodically. From the received beacon packet, each node is able to locally figure out both inbound and outbound link qualities, without exchanging such quality information with neighbors. Another related solution, named blacklisting, can also be found in paper [Gnawali et al. 2004]. Moreover, readers can refer to [Seada et al. 2004][Couto et al. 2003][Yarvis et al. 2002] for more related solutions.

\subsection{Bounded Distance Forwarding}

Bounded Distance Forwarding restricts the distance over which a node can forward a message in a single hop. It is designed as a middleware and can act as an add-on rule to many routing protocols. It provides interfaces for configuring the bounded distance and provides services to filter out all links that are beyond the specified distance. The distance bound is configured based on the degree of radio irregularity of the real devices in a physical system.

We add the Bounded Distance Forwarding rule on the spanning tree module in a vehicle tracking system [He et al. 2004] in which we deploy 60 MICA2 motes. In the experiments, we incrementally increase the single hop forwarding bound from 8 feet to 100 feet and count the number of nodes that report their status and Figure 28 shows this data as a percentage of the total number of nodes deployed. Data points here are average values over five runs.

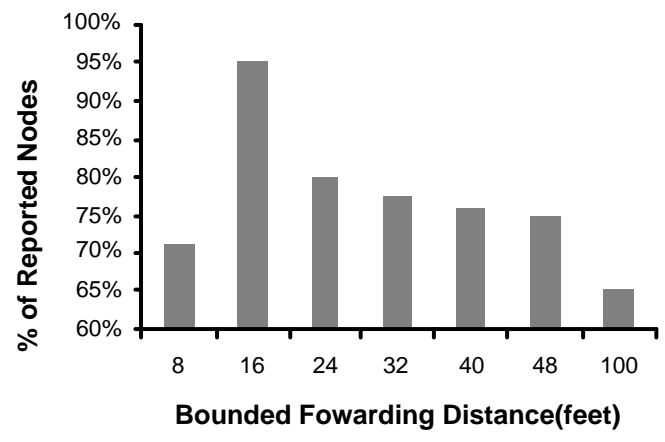

Fig. 28. Performance Evaluation of Bounded Distance Forwarding

Figure 28 indicates two interesting phenomena. First, when we use a very low forwarding bound ( 8 feet) to eliminate the asymmetric links, the performance, however, is not good. This is because relative node density decreases when the enforced communication range is small. Hence, the chance of a network partition increases. Moreover, a smaller forward bound per hop leads to a longer route, thus a higher chance of loss. Second, when the forwarding bound reaches larger values (16 100 feet), link asymmetry becomes the dominating factor. Figure 28 
shows that when the forwarding bound is 16 feet, we receive almost every report. This bound is about half of the MICA2 radio range on the ground. Above 16 feet, performance reduces monotonically because of increase in link asymmetry.

Figure 28 shows that an effective bound, 16 feet here, exists for the application, at which the best performance is achieved. There are generally two methods to get the effective bound for a deployed system. The first method is to tune the bounded distance parameter at the initial deployment of the system, and the second method is to use a feedback control algorithm to converge the bounded distance to the effective value during the runtime of the deployed system.

\subsection{OTHER SOLUTIONS}

In this section, we propose five additional potential solutions to deal with radio irregularity.

-Bidirectional Flooding: The multi-round discovery technique can deal with radio irregularity. However, it needs multiple rounds of flooding to explore different paths, which can be very expensive. In Bidirectional Flooding, the source propagates the RREQ towards the destination through flooding. After the destination receives the RREQ, it propagates the RREP to the source through flooding, instead of using the reverse path along which it received the RREQ from the source. Multi-round discovery cannot guarantee finding symmetry connections within a bounded number of flooding stages. In contrast, bidirectional flooding completes the discovery by flooding twice.

-Learning Function: In an earlier section we mentioned that GF has a higher loss ratio than AODV and DSR, because GF tends to choose the same candidate near the border of its communication range to forward packets to a destination, while AODV and DSR attempt different paths due to the nature of flooding. To address this shortcoming of GF, we can enhance GF with a learning function, which allows a node to make better decisions based on previous routing failures. In the learning function, we distinguish the routing failures arising due to congestion from those that arise due to asymmetric links. This can be done with the help of the 802.11 (DCF) in the MAC layer. If a node receives the CTS, but not the ACK, then the link should be symmetric and the routing failure might be a result of congestion. Such a failure can be solved by retransmissions. However, if a node fails to receive the CTS despite several retransmissions, then the chances are that the link is asymmetric. This learning function allows a node to remember such an asymmetric link and to avoid trying it again before the topology changes.

In a real implementation of this idea, two learning functions are maintained: $F_{\text {link }}$ and $F_{\text {congestion. }}$. Whenever a packet gets lost, whether it is a CTS packet or a DATA packet, both $F_{\text {link }}$ and $F_{\text {congestion }}$ adjust their values according to the current context. For example, if a DATA packet gets lost, $F_{\text {congestion }}$ gets a greater increase than $F_{\text {link }}$, because the CTS was received and there is more chance that congestion, rather than channel quality variation, causes the transmission failure. On the other hand, if the CTS packet gets lost the second time, it is more probable that the channel link quality is bad, and hence $F_{\text {link }}$ gets more increase than $F_{\text {congestion. }}$. By comparing the values of $F_{\text {link }}$ and $F_{\text {congestion }}$, the node decides whether it is congestion or bad link quality that causes the packet 
loss. If $F_{\text {congestion }}>F_{\text {link }}$, the reason is congestion. So backoff and retransmission is a good choice. If $F_{\text {congestion }}<F_{\text {link }}$, the reason is the bad channel quality and rerouting is a better choice. In the case of a tie, a random decision is made between retransmission and rerouting.

To improve the accuracy of the two learning functions: $F_{\text {link }}$ and $F_{\text {congestion }}$, the signal intensity during the carry sensing period can be monitored, together with the packet loss ratio. If both the signal intensity and the packet loss ratio increase, $F_{\text {congestion }}$ gets increased greater than $F_{\text {link }}$. On the other hand, if the signal intensity does not change or even decreases while at the same time the packet loss ratio increases, it is highly probable that the link quality decreases, and $F_{\text {link }}$ gets increased by a larger amount than $F_{\text {congestion }}$.

Actually, the learning function scheme has other applications, besides the application in routing protocols. For example, ESRT [Sankarasubramaniam et al. 2003] is proposed to provide reliable event-to-sink transport service. Nodes monitor local buffer levels. If the routing buffer overflows due to excessive incoming packets, congestion is considered happened, and source nodes in the network are forced to reduce data reporting frequency. Actually, this buffer monitoring scheme does not differentiate whether the buffer overflow is due to congestion and followed by retransmission, or due to the poor link quality of data reporting paths. It is not reasonable for source nodes to reduce data reporting frequencies, if the buffer overflow is caused by the poor routing protocol that chooses poor data reporting paths. The learning function scheme can be used to differentiate these two cases, and choose to either inform source nodes to reduce data reporting frequencies or to inform the routing protocol to choose better data reporting paths.

- RTS Broadcast: Another solution we propose is called the RTS Broadcast, which involves both the MAC and routing layers. We first broadcast a special RTS message, which sets the destination as ANY_NODE. Any node hearing it backs off for a random amount of time and replies with a CTS message. Among all the nodes that send the CTS message, the one that is closest to the destination is chosen as the forwarding candidate. Since the RTS and CTS detect connectivity along the forward and reverse directions of a channel, forwarding packets along asymmetric channels can be avoided.

-High Energy Asymmetry Detection: IEEE 802.11 (DCF) uses a collision-avoidance strategy in which any node upon hearing an RTS, CTS, or DATA message defers its transmission until the data is sent out. However, a node can still interfere with the message transmission even though it is not able to hear any of the RTS, CTS and DATA messages in the presence of asymmetry. The sixth solution we propose is to send out a High Energy Asymmetry Detection (HEAD) control message which has a higher sending power than the other control messages. So more nodes will hear the high-powered signal, and prevent themselves from sending messages. The HEAD message is sent out before the RTS message. Any node other than the destination, upon hearing the HEAD message, sets its NAV to a value large enough so that data can be sent out without contention. The wait time and destination ID are included in the HEAD message. Conflicts may arise if two nodes send out the HEAD messages simultaneously. That is resolved 
in a manner similar to the way to resolve conflicts arising from the simultaneous transmission of two RTS messages. Hence, the transmission sequence is modified from RTS-CTS-DATA-ACK to HEAD-RTS-CTS-DATA-ACK. While the higher sending power of the HEAD message lowers the collision rate, it also introduces an extra control packet, the HEAD packet, which reduces the channel utilization and increases the NAV backoff. The tradeoff between collision rate and desired channel utilization can be balanced by choosing an appropriate value for the sending power.

-Irregularity Insensitive Protocols: There are two avenues for improving protocol performance in the presence of radio irregularity. The first method is to face radio irregularity and avoid getting involved into any trouble brought by radio irregularity. For example, we propose to detect asymmetry links brought by radio irregularity and try to avoid using asymmetry links. The second method is to investigate the assumptions that cause protocol performance to deteriorate in reality and then design protocols that do not make such assumptions. That is, radio irregularity can also be dealt with, by identifying protocol properties that make them particularly insensitive to radio irregularities. For example, the Cricket localization [Priyantha et al. 2000] uses a combination of RF and ultrasound technologies to location devices' locations. Cricket is insensitive to radio irregularity and avoids the problems many localization protocols get involved in because of using the received signal strength to estimate communication distances. The APIT localization protocol [He et al. 2003] is another example that avoids making the ideal radio assumption. Accordingly, irregularity insensitive protocol design is a promising avenue to address the radio irregularity as well as link asymmetry it brings.

Among the eight solutions we put forth above, the last five are still open topics and require further refinements. Extensive analysis and evaluation in the future are required to demonstrate their applicability and effectiveness.

\section{CONCLUSIONS}

In this paper, we confirm the existence of radio irregularity which is the main focus of several recent research papers [Ganesan et al. 2002][Woo et al. 2003][Zhao and Govindan 2003][Cerpa et al. 2003]. Our contributions are as follows:

(1) To the best of our knowledge, our work is the first to bridge the gap between isotropic radio models assumed by most simulators and the anisotropic radio properties found in reality.

After our work was first accepted in MobiSys 2004 [Zhou et al. 2004], an upper layer model [Cerpa et al. 2005] was proposed to simulate link asymmetry in the link layer, without considering the wireless communication detail in the radio layer. We compare our RIM radio model with this link layer model in APPENDIX B.

(2) We propose a novel RIM model that approximates three essential properties exhibited in radio irregularity: anisotropy, continuous variation and difference in sending power. 
(3) We implement the RIM model in GloMoSim, and run a set of simulation experiments to investigate radio irregularity's impact on MAC and routing layer performance. We discover that, among the protocols we evaluate, the radio irregularity has a greater impact on the routing layer than MAC layer. We also discover that radio irregularity has a greater impact on location-based routing protocols than on-demand protocols that use multi-round discovery technique.

(4) We run a set of simulation experiments to investigate radio irregularity's impact on localization and topology control, finding that the increasing radio irregularity leads to larger localization errors, and that the communication connectivity becomes harder to maintain when the radio becomes more irregular.

(5) Finally, we present eight potential solutions. We implement SGF in GloMoSim, and implement the Asymmetry Detection Service and the Bounded Distance Forwarding methods in running systems with 27 60 MICA2 motes. From the data we collect from the simulator and the running system, we find that SGF, Asymmetry Detection Service and Bounded Distance Forwarding greatly improve system performance in the presence of radio irregularity.

The RIM model we put forth in this paper is built based on empirical data collected from MICA2 and MICAZ platforms. So to some degree, this model is self-evaluated. We also conduct preliminary evaluation of the RIM model, in Section 4.2.1, by comparing the degree of radio irregularity between the measured radio pattern from a real device and the radio patterns generated from the RIM model. We are also aware that more extensive performance comparison between the simulated results based on the RIM model and the results from real systems with MICA2 and other devices are needed, to further evaluate the precision of the RIM model. We leave this as future work.

\section{ACKNOWLEDGEMENT}

This work is supported by the National Science Foundation (grant CNS-0435060, grant CCR-0325197, and grant EN-CS-0329609) and by the DARPA IXO offices under the NEST project (grant F336615-01-C-1905). The authors would like to thank the reviewers of ACM Transactions on Sensor Networks for their valuable comments during the revision of this work.

\section{APPENDIX A}

We use the goodness-of-fit statistical testing to determine the statistical distribution of the percentage variance of the path loss (in $\mathrm{dBm}$ ) per degree in the direction that is obtained in our experiments. We find that among different continuous distributions, the Weibull distribution [Devore 1982] has the maximum likelihood of matching our experimental data. A random variable $\mathrm{X}$ that has a Weibull distribution with parameters has a probability density function defined by the following equation, where $a$ is the shape parameter and $b$ is the scale parameter.

Table III shows the likelihood values and the parameters of the Weibull distribution that fits our experimental data. These values are computed at a $95 \%$ confidence level. 
Table III. Data Fitting to the Weibull Distribution

\begin{tabular}{||l|l||l|l||}
\hline & Likelihood & $a$ & $b$ \\
\hline Dataset 1 & 48.55 & 1.13 & 0.28 \\
\hline Dataset 2 & 154.43 & 1.01 & 0.17 \\
\hline Dataset 3 & 145.25 & 0.86 & 0.18 \\
\hline Dataset 4 & 277.44 & 0.67 & 0.16 \\
\hline Dataset 5 & 204.51 & 0.58 & 0.17 \\
\hline Dataset 6 & 111.15 & 0.53 & 0.22 \\
\hline
\end{tabular}

$$
f_{x}= \begin{cases}\left(a / b^{a}\right) \times x^{a-1} \times e^{-\left(\frac{x}{b}\right)^{a}} & \text { if } x \geq 0 \\ 0 & \text { if } x<0\end{cases}
$$

\section{APPENDIX B}

A link layer model for simulating link asymmetry is first proposed in Cerpa's technical report [Cerpa et al. 2004], and later published in IPSN 2005 [Cerpa et al. 2005]. In this model, link asymmetry is simulated without considering the lower layer wireless communications. The RIM model differs with the link layer model in that the RIM model is a radio layer model. The RIM model is proposed to simulate radio irregularity rather than link asymmetry, which happens to be one result of radio irregularity reflected in the link layer. Since the RIM model incorporates details in radio communication, it can address more issues that the simpler link layer model can not simulate. We illustrate four of them as follows:

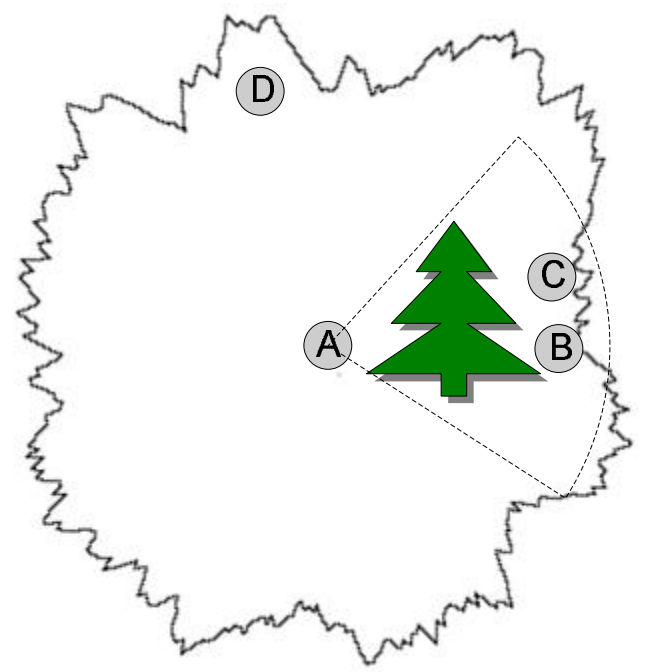

Fig. 29. Link Qualities in Adjacent Directions

First, the RIM protocol can simulate the phenomenon that links in very similar directions from the same transmitter have similar link qualities. As shown in Figure 29, there is a big tree, in the east direction of transmission node A. So the 
signal from A suffers more path losses, due to the tree, in the directions specified by the fan area compared with other directions. For example, when A's signal propagates to $\mathrm{B}$ and $\mathrm{C}$, it suffers similar path losses. But the signal suffers less path loss when it propagates from A to D. Accordingly, transmitter A has similar link qualities with B and C. The link layer model does not simulate directionality of signal propagation and this phenomenon is not addressed. However, our RIM model can reflect this fact, because all the $k_{i}$ values in adjacent directions are related in the sense that $k_{i+1}$ is calculated based on $k_{i}$.

Second, the RIM model can be used to study the impact of radio irregularity on localization protocols that is sensitive to radio patterns. In section 7 of this paper, we study the impact of radio irregularity on the Centroid algorithm as an example. In a similar way, our model can be use to study the impact of radio irregularity on many other localization protocols, which use the received signal strength indicator to help location decisions. However, the link layer model that does not simulate the radio communication process can not be used for this study.

Third, the link layer model does not regenerate radio signals, so they can not really simulate radio interference, which also has a significant effect on link qualities. The RIM model works in the radio layer, which uses mature simulation techniques, such as TWO-RAY model and RICIAN mode [Shankar 2001] implemented in GloMoSim, to simulate the radio propagation and fading in a specified direction. And the DOI and VSP parameters proposed in RIM are used to account for the irregularity in different directions as well as hardware differences. Accordingly, the RIM model can also simulate radio interference, which also leads to decreased link qualities.

Fourth, with the DOI and VSP parameters, the RIM model can simulate radio irregularity due to the two root causes we found in our sensor device experiments: the path loss differences and the power heterogeneity. This offers the users the ability to configure different DOI and VSP values according to their specific hardware properties and deploy environment, to simulate the system performance within the specific hardware and environment context. However, the link layer model is not able to differentiate which of the two root causes leads to the decreased link quality and how much each of them contributes.

On one hand, we acknowledge that the link layer model has a higher abstraction and is hence smaller and light weight. On the other hand, by simulating wireless communication details, the RIM radio model is more powerful, and able to address more issues that are beyond the ability of the link layer model.

\section{REFERENCES}

Bahl, P. and Padmanabhan, V. N. 2000. RADAR: An In-Building RF-Based User Localization and Tracking System. In IEEE InfoCom 2000.

Battery Lifetime. Battery Technology Life Verification Test Manual. http://www.uscar.org/consortia\&teams/USABC/Manuals/Technology Life Verification Test Manual - Feb 2005.pdf.

Bharghavan, V., Demers, A., Shenker, S., and Zhang, L. 1994. MACAW: A Media Access Protocol for Wireless LANs. In ACM SigComm 1994. 212-225.

Cerpa, A., Busek, N., and Estrin, D. 2003. SCALE: A Tool for Simple Connectivity Assessment in Lossy Environments. Tech. rep., CENS Technical Report 0021. 
Cerpa, A. and Estrin, D. 2002. ASCENT: Adaptive Self-Configuring Sensor Networks Topologies. In IEEE InfoCom 2002.

Cerpa, A., Wong, J. L., Kunng, L., Potkonjak, M., and Estrin, D. 2004. Statistical Model of Lossy Links in Wireless Sensor Networks. Tech. rep., CENS Technical Report 0041.

Cerpa, A., Wong, J. L., Kuang, L., Potkonjak, M., and Estrin, D. 2005. Statistical Model of Lossy Links in Wireless Sensor Networks. In ACM/IEEE IPSN'05.

Chen, B., Jamieson, K., Balakrishnan, H., and Morris, R. 2001. SPAN: An Energy-Efficient Coordination Algorithm for Topology Maintenance in Ad-Hoc Wireless Networks. In ACM MobiCom 2001.

ChipconCC1000. Chipcon CC1000 Low Power Radio Transceiver. http://www.chipcon.com.

ChipconCC2420. CC2420 2.4 GHz IEEE 802.15.4 / ZigBee-ready RF Transceiver. http://www.chipcon.com.

Cormen, T. H., Leiserson, C. E., Rivest, R. L., and Stein, C. 2002. Introduction to Algorithms. The MIT Press.

Couto, D. S. J. D., Aguayo, D., Bicket, J., And Morris, R. 2003. A High-Throughput Path Metric for Multi-Hop Wireless Routing. In ACM MobiCom 2003.

CROSSBOW. XBOW Mote Specifications. http://www.xbow.com.

Dam, T. and K. Langendoen. 2003. An Adaptive Evergy-Sufficient MAC Protocol for Wireless Sensor Networks. In ACM SenSys 2003.

Devore, J. L. 1982. Probability and Statistics for Engineering and the Sciences. Brooks/Cole Publishing.

Ganesan, D., Krishnamachari, B., Woo, A., Culler, D., Estrin, D., and Wicker, S. 2002. Complex Behavior at Scale: An Experimental Study of Low-Power Wireless Sensor Networks. Tech. rep., Technical Report UCLA/CSD-TR 02-0013.

Gnawali, O., Yarvis, M., Heidemann, J., and Govindan, R. 2004. Interaction of Retransmission, Blacklisting, and Routing Metrics for Reliability in Sensor Network Routing. In IEEE SECON 2004.

He, T., Huang, C., Blum, B. M., Stankovic, J. A., And Abdelzaher, T. F. 2003. Range-Free Localization Schemes in Large Scale Sensor Networks. In ACM MobiCom 2003.

He, T., Krishnamurthy, S., Stankovic, J. A., Abdelzaher, T. F., Luo, L., Stoleru, R., Yan, T., Gu, L., Hui, J., ANd Krogh, B. 2004. Energy-Efficient Surveillance System Using Wireless Sensor Networks. In ACM MobiSys 2004. 270-283.

He, T., Stankovic, J. A., Lu, C., And Abdelzaher, T. F. 2003. SPEed: A Stateless Protocol for Real-Time Communication in Sensor Networks. In IEEE ICDCS 2003.

Hightower, J., Boriello, G., And Want, R. 2000. SpotON: An Indoor 3D Localization Sensing Technology Based on RF Signal Strength. Tech. rep., University of Washington CSE Report 2000-02-02.

Hightower, J., Vakili, C., Borriello, G., , And Want, R. 2000. Design and Calibration of the SpotON Ad-Hoc Location Sensing System. Tech. rep., unpublished. August.

Hoff, B. and Azuma, R. 2000. Autocalibration of an Electronic Compass in an Outdoor Augmented Reality System. In Proceedings of IEEE and ACM International Symposium on Augmented Reality 2000. 159-164.

IEEE 802.11 1999. IEEE 802.11, part II: Wireless LAN Medium Access Control (MAC) and Physical Layer (PHY) Specification. ANSI/IEEE Std. 802.11, 1999.

IEEE 802.15.4 1999. IEEE 802.15.4, Wireless Medium Access Control (MAC) and Physical Layer (PHY) Specifications for Low-Rate Wireless Personal Area Networks (LR-WPANs). IEEE Std. 802.15.4, 2003.

Intanagonwiwat, C., Govindan, R., and Estrin, D. 2000. Directed Diffusion: A Scalable and Robust Communication Paradigm for Sensor Networks. In ACM MobiCom 2000. 56-67.

Johnson, D. B. And Mattz, D. A. 1996. Dynamic Source Routing in Ad Hoc Wireless Networks. In Mobile Computing, edited by Tomasz Imielinski and Hank Korth, chapter 5, pages 153-181. Kluwer Academic Publishers, 1996. 
KARn, P. 1990. MACA - A New Channel Access Method for Packet Radio. In $A R R L / C R R L$ Amateur Radio 9th Computer Networking Conference. 134-140.

KARP, B. 2000. Geographic Routing for Wireless Networks. Ph.D. thesis, Harvard University, Cambridge, MA.

Karp, B. And Kung, H. T. 2000. GPSR: Greedy Perimeter Stateless Routing for Wireless Networks. In ACM MobiCom 2000. 243-254.

Kleinrock, L. And Tobagi, F. A. 1975. Packet Switching in Radio Channels: Part I - Carrier Sense Multiple-Access Modes and their Throughput-Delay Characteristics. IEEE Transactions on Communications COM-23, 1400-1416.

Ko, Y.-B. And Vaidya, N. H. 1998. Location-Aided Routing (LAR) in Mobile Ad Hoc Networks. In ACM MobiCom 1998. 66-75.

N. Bulusu, J. H. And Estrin, D. 2000. GPS-Less Low Cost Outdoor Localization for Very Small Devices. IEEE Personal Communications Magazine, 28-34.

Niculescu, D. And Nath, B. 2003. DV Based Positioning in Ad hoc Networks. Journal of Telecommunication Systems.

Perkins, C. E. And Royer, E. M. 1999. Ad-hoc On-Demand Distance Vector Routing. In The Second IEEE Workshop on Mobile Computing Systems and Applications. 90-100.

Polastre, J., Hill, J., And Culler, D. 2004. Versatile Low Power Median Access for Wireless Sensor Networks. In ACM SenSys 2004.

Priyantha, N. B., Chakraborty, A., and Balakrishnan, H. 2000. The Cricket LocationSupport System. In ACM MobiCom 2000.

Rajendran, V., Obraczka, K., And Garcia-Luna-Aceves, J. 2003. Energy-Efficient, CollisionFree Medium Access Control for Wireless Sensor Networks. In ACM SenSys 2003.

RF Chamber. Turnkey Chambers. http://www.tdkrfsolutions.com/chambers.htm.

Sankarasubramaniam, Y., Ö. B. Akan, and Akyildiz, I. F. 2003. Esrt: event-to-sink reliable transport in wireless sensor networks. In ACM MobiHoc '03. 177-188.

Seada, K., Zuniga, M., Helmy, A., and Krishnamachari, B. 2004. Energy-Efficient Forwarding Strategies for Geographic Routing in Lossy Wireless Sensor Networks. In ACM SenSys 2004.

Shankar, P. M. 2001. Introduction to Wireless Systems. John Wiley and Sons, Inc.

Whitehouse, K. And Culler, D. 2002. Calibration as Parameter Estimation in Sensor Networks. In ACM International Workshop on Wireless Sensor Networks and Applications.

Woo, A. And Culler, D. 2001. A Transmission Control Scheme for Media Access in Sensor Networks. In ACM MobiCom 2001.

Woo, A., Tong, T., And Culler, D. 2003. Taming the Underlying Challenges of Reliable Multihop Routing in Sensor Networks. In ACM SenSys 2003.

Xu, Y., Heidemann, J., And Estrin, D. 2001. Geography-Informed Energy Conservation for Ad Hoc Routing. In ACM MobiCom 2001.

Yan, T., He, T., And Stankovic, J. A. 2003. Differentiated Surveillance for Sensor Networks. In ACM SenSys 2003.

Yarvis, M. D., Conner, W. S., Krishnamurthy, L., Mainwaring, A., Chhabra, J., and ElLIOTT, B. 2002. Real-World Experiences with an Interactive Ad Hoc Sensor Network. In IWAHN 2002.

Ye, W., Heidemann, J., And Estrin, D. 2002. An Energy-Efficient MAC Protocol for Wireless Sensor Networks. In IEEE Info Com. 1567-1576.

Zeng, X., Bagrodia, R., And Gerla, M. 1998. GloMoSim: A Library for Parallel Simulation of Large-Scale Wireless Networks. In The 12th Workshop on Parallel and Distributed Simulations.

Zhao, J. And Govindan, R. 2003. Understanding Packet Delivery Performance in Dense Wireless Sensor Networks. In ACM SenSys 2003.

Zhou, G., He, T., Krishnamurthy, S., And Stankovic, J. A. 2004. Impact of Radio Irregularity on Wireless Sensor Networks. In ACM MobiSys 2004. 125-138. 